\title{
Bollettino di Islamistica
}

\author{
Roberto Tottoli \\ Università degli Studi di Napoli "L’Orientale" \\ rtottoli@unior.it
}

\section{Origini dell'islam}

Tra le innumerevoli opere che trattano delle origini dell'islam evocando la Tarda Antichità, il lavoro di Olof Heilo è sicuramente originale. ${ }^{1}$ Il punto di partenza è la prospettiva della classicità tardo-antica e quindi del ruolo e declino di Costantinopoli a cavallo del sesto e settimo secolo e l'impatto su questa realtà della novità portata dall'Islam. L'Autore analizza questo passaggio storico non per discernere teologia da storia, bensì per ricostruire quelle linee di pensiero che hanno attraversato una parte e l'altra, e poi permesso grandi sovvertimenti e profonde continuità tra un prima e un dopo. Su questi presupposti si formula quindi l'ipotesi che l'Islam sia stato un fenomeno consonante con le tensioni latenti nella regione, anche sul versante bizantino, tra potere imperiale e aspettative apocalittiche/escatologiche. Ne consegue che il periodo che vide l'origine dell'Islam diviene un'età aperta al confronto e fruttuosa di reciproche influenze e partecipazioni, in un quadro regionale più ampio che prevale su formali aspetti confessionali. Tutte le ipotesi sono interessanti e il saggio è anche brillante nell'esposizione e nell'attraversare soprattutto testimonianze e letteratura da parte bizantina per costruire le coordinate di un rapporto interconfessionale alle origini del mondo islamico. Certo è che, tanto più fascinoso pare tutto il costrutto, tanto più ci si allontana da testi e da testimonianze storiche, e il saggio diviene un quadro interpretativo che si apre a più di un dubbio, senza poter, ovviamente, superare la profonda frattura con chi tenta una ricostruzione storica su dati originati da teologia e discorso religioso. Non sia questo a demerito di questo agile saggio, che sconta a tal riguardo la complessità di una questione che continuamente interroga studiosi e ricercatori.

1 Heilo, O. Eastern Rome and the Rise of Islam. "Culture and Civilization in the Middle East" n. 51, London-New York, Routledge, 2016, xii + 149 p. 


\section{Corano ed esegesi coranica}

Lo studio delle figure profetiche nel Corano viene oggi in genere, e a ragione, separato dalle riletture esegetiche e tradizionali successive. Nel caso della monografia su Noè di Carlos A. Segovia, ad esempio, ciò serve ad affrontare per un caso specifico il tema dell'intertestualità e del dialogo del testo coranico con le culture religiose dell'area vicino-orientale, in particolare con la letteratura cristiana. ${ }^{2}$ È del resto questa una direttrice ben precisa negli studi coranici più recenti, variamente legata ai percorsi di indagine di Gabriel Said Reynolds. Segovia offre un'introduzione completa e schematica degli studi precedenti su Noè coranico, sottolineando come i diversi e numerosi percorsi di indagine hanno spesso sottovalutato le possibilità di vero dialogo delle versioni coraniche con la letteratura coeva, in particolare con le rielaborazioni di apocalissi legate anche alla figura di Noè. L'analisi non può che partire dai brani coranici dedicato a Noè e dai processi di riscrittura che il testo preserva, e quindi la valutazione che di ciò se ne fa. Nel caso di Segovia non si tratta di un'evoluzione dettata da peculiarità del testo coranico in sé, quanto piuttosto di una ricezione e rielaborazione di motivi circolanti tra giudaismo e primo cristianesimo, che nulla toglie all'originalità coranica, bensì lo colloca in fruttuoso dialogo con le culture circostanti. Gli elementi messianici e le peculiarità del Noè coranico sono poi viste in relazione a questo ambiente formativo. La portata critica di tale assunto non sfugge già dall'introduzione in cui si dà riscontro alle divisioni e contrapposizioni nella ricerca contemporanea sul Corano. Tale saggio ha di conseguenza una duplice funzione di cui si deve dar merito al suo Autore: porta un ulteriore tassello nel mosaico sempre più vivido degli studi coranici di oggi e offre un'analisi esaustiva dei passi coranici su Noè, indagando nel dettaglio nelle loro caratteristiche formali e di contenuto.

Gerhard Böwering e Yousef Casewit hanno edito il commentario minore di Ibn Barrağān (m. 1141), accompagnando l'edizione con un'introduzione su Autore e opera e quindi dai necessari apparati di supporto al testo. ${ }^{3}$ L'esegesi coranica di Ibn Barrağāan riflette l'originalità del suo pensiero e le problematiche di una lettura complessa, tra suggestioni di ogni tipo e in ogni direzione, in

2 Segovia, C.A. The Quranic Noah and the Making of an Islamic Prophet. A Study of Intertextuality and Religious Identity Formation in Late Antiquity. "Judaism, Christianity, and Islam-Tension, Transmission, Transformation" n. 4, Berlin-Boston, De Gruyter, 2015, $\mathrm{xvi}+154 \mathrm{p}$.

3 A Qửān Commentary by Ibn Barrajān of Seville (d. 536/1141). Ị̇āḥ al-ḥikma bi-aḥkām al-ibra (Wisdom Deciphered, the Unseen Discovered. A cura di G. Böwering e Y. Casewit. "Texts and Studies on the Qur’ān" n. 10, Leiden—Boston, Brill, 2016, xi + 956 (892 in ar.) p. 
un periodo particolarmente fecondo eppure attraversato da spinte e movimenti in ogni direzione. Ibn Barrağān in particolare, viene descritto come esperto di hadìt oppure di kalām e di letture coraniche, a ben rappresentare le molteplici competenze dell'esperto in letteratura e tradizioni religiose musulmane nel Medioevo islamico. Si tratta, quindi, di un commentario sui generis, ad esempio nella scelta dei versetti commentati, ma non per questo meno significativo e utile per la storia del genere e della letteratura della Spagna musulmana. Terminologia e argomenti trattati, come ben evidenziato dall'introduzione, sono originali, così come una serie di concezioni, ad esempio la cosmogonia, o quelle chiaramente influenzate da teorie ismailite o dall'enciclopedia degli Ihwān al-șafã. L'assenza di riferimenti espliciti, suggeriscono i curatori, potrebbe proprio essere collegata a prudenza e attenzione nel non usare fonti contestabili e che ne avrebbero attirato critiche e accuse. L'edizione è condotta sostanzialmente su tre manoscritti (Mahmud Pasa 3 e 4, e Ms Murat Molla 35), prendendo i Pasa 3 e 4 come base e utilizzando l'altro per segnalare diverse lezioni utili e con rari interventi, congetturali, di correzione. Particolarmente interessante è la citazione costante di hadìt nel commentario che riflette l'interesse di Ibn Barrağān, riflesso in altra sua opera (al-Iršād ilà subul al-rašād), per l'analisi del rapporto tra letteratura dei detti di Muḥammad e la sua concordanza con i contenuti del Corano (in particolare sui punti di convergenza tra il Corano e gli ḥadīt riportati da Muslim (m. 875) nel suo Șaḥiḥ).

Lattività esegetica, soprattutto in età contemporanea, spesso si sovrappone a quella di traduzione del testo coranico in aree non arabofone. Suha TajiFarouki ha curato una raccolta di saggi unica per varietà di realtà indagate e per autori e opere su questi temi analizzati. ${ }^{4} \mathrm{~S}$. Taji-Farouki è tuttavia ben più di una curatrice. La sua introduzione è ampia e ricca di dati, e con le sue oltre cento pagine già costituisce una sorta di piccola monografia sul tema della traduzione e dell'esegesi in età contemporanea. Oltre a questo ha contribuito con un saggio e altri due scritti in collaborazione con altri autori dei dieci. Nella maggior parte dei casi si analizzano singole figure di esegeti e/o traduttori/ commentatori del Corano, in aree quali Bosnia, Turchia, passando poi alle produzioni in urdu, malay, swahili, quindi in Iran e Cina. Le incursioni in produzioni occidentali, in particolare negli Stati Uniti e in Germania, trattano di un autore nel primo caso (Muhammad al-Āṣī) e di un tema nel secondo (le

4 The Qur'an and Its Readers Worldwide. A cura di S. Taji-Farouki. "Qur'anic Studies Series", n. 14, London, Oxford University Press in collaboration with The Institute of Ismaili Studies, xix +633 p., con introduzione della Curatrice e contributi di A. Karić e S. Taji-Farouki, I. Albayrak, M. Mir, A.H. Johns e S. Taji-Farouki, T. Zadeh, S. Naguib, S. Taji-Farouki, F. Topan, A. Christmann, M. Dillon. 
interpretazioni di Cor. 4:34). Solo il capitolo di S. Naguib, non meno interessante, affronta una figura di esegeta più nota degli altri e che ha prodotto in arabo: 'Â’išah 'Abd al-Raḥmān, alias Bint al-Š̄ṭi' (1913-1998). Tutta la raccolta ha il merito di ampliare in modo inaspettato l'ambito degli studi sull'esegesi contemporanea e di contribuirvi in un settore significativo come quello del rapporto tra attività esegetica e traduzione per i non arabofoni che costituiscono la maggioranza dei musulmani. Al di là delle considerazioni sulla ammissibilità di traduzioni del Corano, la realtà del xx secolo e di oggi mostra in particolare come nel gioco tra esegesi e tradizione interpretativa si apre uno spazio sconfinato per una ridiscussione e interpretazione del testo sacro in ogni luogo del mondo islamico. Si tratta di una ridiscussione che attraversa tutta la storia islamica, e che emerge con modalità ancor più complesse e ricche nella realtà di oggi, come questo lavoro introduce, mostra e discute in modo rimarchevole.

Nella storia dell'esegesi islamica Ibn Ğarīr al-Ṭabarī (m. 923) occupa un ruolo centrale. Un numero delle rivista Journal of Qur'anic Studies, curato da Marianna Klar, discute del suo commentario attraverso punti di vista e approcci diversi. ${ }^{5}$ I cinque contributi condividono l'analisi di un concetto o tema specifico affrontato nell'opera e valutato alla luce della storia esegetica e tradizionale islamica dei primi tre secoli. In particolare la storia dell'uccisione di Husayn è analizzata da T. Hylén, l'organizzazione della storia della caduta di Adamo da M. Klar e il concetto di huğğah da D. Stewart. W. Saleh procede invece a una lettura del commentario di Țabarī attraverso lo "specchio" delle Ta'wìlāt ahl al-sunnah di al-Māturīdī, mentre U. Mårtensson discute della concezione del Corano nel complesso dell'opera di Tabarī. Tutti i capitoli sono solidi esempi di ricerca condotta sui testi e quindi su quello che le fonti scritte possono offrire, pur in piena consapevolezza della retorica dello scritto e dei limiti imposti dal genere letterario. Nel loro complesso, essi dimostrano, anche quando i risultati che vengono offerti sembrano prendere direzioni diverse, la ricchezza della costruzione letteraria dell'autore Țabarī e del suo commentario nel panorama della letteratura islamica e del genere esegetico. Non vi sono dubbi che il suo contributo nella definizione di concetti rappresenti uno snodo significativo nel passaggio dai primi tre secoli di produzione tradizionale all'attività letteraria successiva, eppure il complesso dei saggi dimostra anche molto altro. La costruzione dell'autore Țabarī e le sue contingenze politiche e aspirazioni personali definiscono una serie di prese di posizione e di concezioni che sono assai più complesse dei materiali precedenti e che, allo stesso tempo,

5 "Exegetical facets of Muhammad b. Jarīr al-Ṭabarī (d. 310/923)". A cura di M. Klar. Journal of Qur'anic Studies, 18, 2 (2016), v + 218 p., con premessa della Curatrice e contributi di U. Mårtensson, T Hylén, M. Klar, D.J. Stewart, W.A. Saleh. 
non dovrebbero essere più definite espressione di classicità. Lo dimostrano U. Mårtensson e soprattutto W. Salih, evidenziando come il commentario rifletta scelte personali, giustificate, e una selettività che non sempre corrisponde alla tradizione esegetica e che, perciò, non va affatto considerata normativa o mainstream. Così facendo, non se ne ridimensiona il ruolo, ma se ne accresce specificità e anche significato, in un'opera che, va ricordato, ha goduto di alterne fortune nel corso della storia islamica dove non ha comunque mai avuto questo ruolo centrale né tantomeno normativo.

L'opera di Ḥārit b. Asad al-Muhāsibī (m. 857) include un Kitāb fahm alQurāan che, benché non si tratti di un commentario vero e proprio, appartiene senz'altro alla letteratura esegetica. L'edizione commentata dall'unico manoscritto, la sua traduzione e lo studio specifico è l'argomento della monografia di Berenike Metzler. ${ }^{6}$ L'opera non è inedita dato che era già stata pubblicata nel 1971 da Ḥusayn Quwwatlī insieme all'edizione del Kitāb mä̉iyyat al-'aql dello stesso al-Muhāsibī. Il lavoro della Metzler è tuttavia ben diverso e parte dal testo, che corregge laddove necessario dandone puntuale riferimento in nota, accompagnato a fronte da una traduzione annotata. Un'introduzione e una parte finale commentano e discutono le parti in cui viene suddiviso il testo che discute di vari aspetti dell'esegesi coranica, dalla recitazione all'abrogazione, passando per questioni di tipo teologico e quindi di approccio al testo sacro. Lo studio risulta particolarmente accurato e felice nella lettura che offre dell'opera e nella ricostruzione, tramite ciò, dell'attività di Muhāsibī che fu un autore particolarmente significativo anche per originalità della sua opera. In particolare, l'Autrice analizza la sua posizione nella realtà storica e religiosa della prima metà del nono secolo, segnata da forti contrapposizioni proprio di natura teologica e tradizionale che portarono alla mihnnah e quindi al finale trionfo del tradizionalismo. L'opera in questione evidenzia in modo chiaro come Muhāsibī debba essere considerato una sorta di semi-razionalista e come la ricezione e dialettica con il mu'tazilismo ne caratterizzi approccio e temi scelti nella sua breve introduzione alla comprensione del Corano. Questo posizionamento è senza dubbio ciò che ne determina la relativa originalità ma anche la difficoltà di collocazione nei confronti dello stesso mu'tazilismo e di Ahmad b. Hanbal nello schieramento avverso, che in qualche modo polarizzarono, anche nelle ricostruzioni storiche, i termini dello scontro. Al di là dei problemi di collocazione in questa polarizzazione, posizioni intermedie come quella di Muhāsibī furono quelle alla lunga destinate ad avere un ruolo significativo e importante, ad esempio nelle nascenti scuole teologiche (linee

6 Metzler, B. Den Koran verstehen. Das Kitāb Fahm al-Qurān des Hāriț b. Asad al-Muhāàibī. "Diskurse der Arabistik" n. 22, Wiesbaden, Harrassowitz Verlag, 2016, xi + 377 p. 
di sviluppo dell'aš‘arismo) e anche per la sensibilità sufi, di cui Muhāsibī viene giustamente considerato una sorta di precursore.

\section{Tradizioni islamiche}

Pavel Pavlovich ha prodotto il lavoro più significativo, dopo quelli di Harald Motzki, sulla letteratura di hadiț..7 Il tema scelto è quello, lungamente e assai dibattuto, del termine kalälah, ovvero il problema del suo significato in Cor. 4:12 e tutte le questioni sollevate dai detti di Muhammad che ne accennano. Il punto di partenza sono gli studi di David Powers sul Corano e quelli di Agostino Cilardo sulle tradizioni, ma l'Autore va ben oltre. Innanzitutto offre un'esaustiva introduzione critica sullo stato dell'arte degli studi su questa letteratura, evidenziandone limiti e problemi irrisolti. Dall'altro mostra la potenzialità dei nuovi mezzi informatici per analizzare la variantistica dei materiali trasmessi, facendo quindi fare un passo ulteriore alla tecnica cosiddetta isnād-cum-matn per tentare una datazione plausibile. Anche qui, tuttavia, Pavlovich compie la necessaria distinzione tra autenticità e storicità, e mostra consapevolezza e prudenza oltre che una solida logica nel render conto dei problemi che le varie versioni attestate evidenziano. Il saggio rappresenta, anche nelle sue notevoli dimensioni, la dimostrazione più aggiornata dalle possibilità di questo metodo, rese ancora più complesse dai dati resi disponibili da data-base e programmi informatici che permettono oggi un'analisi quantitativa notevole. Ciò è ovviamente preliminare all'analisi qualitativa e all'acume necessario per orientarsi tra versioni diverse, protagonisti all'opera segnalati negli isnād e provenienze regionali, il tutto sempre messo alla prova tra plausibilità storica $\mathrm{e}$ falsificazione o corruzione. Nel caso specifico, le divergenze interpretative, che sono collegate alle diverse occorrenze coraniche, vengono identificate come frutto di interpretazioni diverse nate in ambiti regionali e temporali diversi. Resta un solo dubbio, pensando a ricerche tradizionali e anche alle critiche espresse da altri per altri temi e studi, ovvero che il prodotto finale di tale montagna di dati non si discosta troppo da ciò che studi esclusivamente sui contenuti e dal combinato di questi hanno sempre sostenuto, ovvero che elementi di storicità emergono a partire dall'ultimo quarto del I secolo dell'egira (700 ca.) e che le evoluzione successive sono connesse a svariati fattori che implicano motivazioni di vario tipo.

7 Pavlovich, P. The Formation of the Islamic Understanding of kaläla in the Second Century AH (718-816 CE). Between Scripture and Canon. "Islamic History and Civilization. Studies and Texts" n. 126, Leiden-Boston, Brill, 2015, xviii + 577 p. 
Christian Lange è senza dubbio lo studioso che ha scritto o curato i contributi più importanti degli ultimi anni sull'escatologia musulmana. Il suo saggio su paradiso e inferno, però, per certi versi li riassume e anche li supera per completezza di informazione e respiro generale. ${ }^{8}$ Non manca nulla in questo ritratto delle tradizioni relative al paradiso e all'inferno islamici: una ampia rassegna di fonti islamiche di ogni genere, una lettura comparativa dove necessaria e la giusta sensibilità nel percorrere vie tortuose tra dati coranici, prime tradizioni (hadīt etc.) e le produzioni letterarie che le hanno raccolte. I capitoli definiscono in modo preciso tutte le questioni. Una necessaria premessa metodologica e di rassegna degli studi precedenti non manca di profili esemplificativi altrettanto importanti. L'analisi dei passi coranici percorre immaginario, dati quantitativi e ipotesi legate alla datazione e cronologia della rivelazione. La stessa attenzione e capacità analitica è riservata alle tradizioni più antiche, al loro formarsi e al loro divenire letteratura. In quest'ultimo caso, l'Autore riesce laddove è sempre assai problematico orientarsi, ovvero definire peculiarità autoriali in una letteratura basata su citazioni o omissioni di materiali precedenti. Questo profilo di storia letteraria si conclude con l'analisi di opere di al-Suyūṭī (m. 1505) e al-Maǧlisī (m. 1699). La seconda parte del saggio tocca invece le tradizioni su inferno e paradiso negli altri ambiti di elaborazione del discorso religioso, non necessariamente estranei al costrutto tradizionale finora delineato, ma connessi a questioni specifiche, quali i dibattiti teologici e filosofici, le visione sciite, le interpretazioni mistiche e poi le rappresentazioni delle dimore eterne in topografia, architettura e rituale. Un breve epilogo arriva all'età contemporanea, completando così un quadro esaustivo basato su una rassegna esaustiva di letteratura. Questo è forse il dato più caratteristico e che va salutato con la giusta enfasi: un approccio sofisticato, attento a questioni metodologiche e quindi di lettura al di là della retorica dei testi, e sempre consapevole delle implicanze storico-religiose, è servito da un'acribia rara e da una capacità di lettura ammirevole. Il prodotto finale è per certi versi un saggio unico, che ricorda il meglio della vecchia scuola islamistica, con in più la visione ampia più tipica dei prodotti contemporanei.

La credenza nei ǧinn è un aspetto fondamentale delle concezioni islamiche, fin da Corano e, di conseguenza, nella tradizione successiva. Tobias Nünlist ne offre un ritratto ad ampio raggio che aspira a completezza ed esaustività per le varie questioni toccate. ${ }^{9}$ La premessa chiarisce quanto esplicitato nel

8 Lange, C. Paradise and Hell in Islamic Traditions. New York, Cambridge University Press, 2016, xvii +365 p.

9 Nünlist, T. Dämonenglaube im Islam. Eine Untersuchung unter besonderer Berücksichtigung Schriftlicher Quellen aus der vormodernernen Zeit (600-1500). "Studies in the History and Culture of the Middle East", n. 28, Berlin—Boston, Walter de Gruyter, 2015, xiii + 608 p. 
sottotitolo, ovvero il proposito di analizzare la produzione letteraria premoderna per ricavarne la definizione delle credenze musulmane nei ğinn, avvertendo però che questa "grande" tradizione deve essere misurata con la "piccola" tradizione che emerge dalla osservazione etnografica e che registra la pratica vissuta. Inoltre, il saggio fa costante riferimento alla produzione inedita lasciata a Basilea da Fritz Meier (m. 1998): una mole ingente di materiali raccolti che avrebbe dovuto confluire in opere poi non realizzate, tra cui anche sull'argomento specifico dei ğinn. I vari capitoli del saggio offrono poi un'analisi sistematica delle varie questioni che le fonti scritte mettono in evidenza. Si parte dalla definizione della metodologia, la questione dell'esistenza dei ğinn in alcune opere classiche di vario genere, e si giunge alle varie tipologie di ğinn descritte dalle fonti, la loro religione e il loro ruolo in varie direzioni. La parte finale discute di Salomone e di come i ğinn giochino un ruolo anche se all'interno di una tradizione profetica che viene letta nella sua complessità storico-religiosa in maniera efficace. Non vi sono dubbi che tale lavoro copre un vuoto, uno dei tanti, che riguardano concetti-base del credo musulmano. Le questioni di fondo, quali il rapporto tra contenuti coranici e credo pre-islamico, oppure il rapporto tra generi letterari nel trattare dei ğinn, sono costantemente presenti ed evocati. La consapevolezza metodologica, pienamente condivisibile, è inoltre servita da un'efficace capacità di lettura delle fonti e da un profilo complesso che è fondamentalmente riuscito e riesce a cogliere i temi più importanti all'interno di una scelta di fonti significative. L'unico punto su cui ci permettiamo tuttavia di eccepire riguarda proprio la scelta delle fonti, tra

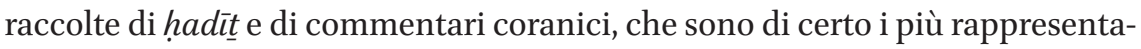
tivi e utilizzati, ma che oggi, in piena età digitale, paiono riduttivi. È evidente che ciò è frutto di una condivisibile scelta di "lettura" delle opere, alla ricerca anche del loro orientamento, e non di estrapolazione di passi che menzionino i ğinn. Eppure perché precludersi le possibilità offerte dai data-base per ricercare anche una maggiore esaustività, tanto più indagando in un argomento che si prestava alla ricerca per lemmi? Questo è l'unico dubbio per un lavoro di notevole valore.

Feryal Salem affronta un autore centrale per gli sviluppi di alcune tematiche, e correlata diffusione di tradizioni, nel primo islam come 'Abd Allāh b. al-Mubārak (m. 797).$^{10}$ Il saggio affronta tutte le principali questioni connesse alla sua complessa personalità e alle sue opere sul ğihād, sullo zuhd e quindi ai materiali nella letteratura di hadìt che gli vengono attribuiti. Il tutto è

10 Salem, F. The Emergence of Early Sufi Piety and Sunnī Scholasticism. 'Abdallāh b. alMubārak and the Formation of Sunnī Identity in the Second Islamic Century. "Islamic History and Civilization. Studies and Texts" n. 125, Leiden-Boston, Brill, 2015, vii + 165 p. 
preceduto da un inevitabile capitolo sulla sua biografia, che mette in luce soprattutto i suoi viaggi di studio e raccolta di tradizioni, e la sua partecipazione all'azione diretta al confine bizantino. Vi è molto, in tutto ciò, di storia del primo islam, sull'origine della diffusione e trasmissione della tradizione e letteratura religiosa e di costruzione a posteriori di una biografia notevole, il tutto mescolato e rielaborato in maniera non sempre facile da delineare. L'introduzione un po' scolastica e semplicistica evidenzia alcuni limiti nel ritratto che viene offerto dall'Autrice, ovvero quello di riprodurre, più agnosticamente che acriticamente, quanto riportano in proposito le fonti arabe. Nulla di male in ciò, ma la deliberata assenza di una chiave interpretativa non è certo la panacea per confrontare approcci assai diversi negli studi sull'islam delle origini e nasconde il fatto che cautela e attenzione critica sono comuni sia a chi ha un approccio possibilistico alle fonti arabe e chi invece le rigetta in toto. Con queste premesse, il saggio risulta un'utile introduzione a una figura centrale del primo islam, che non risolve però gran parte delle questioni che tratta relative alla sua biografia, ai suoi viaggi in cerca di hadiț, alla sua opera e al rapporto tra biografia e opera, ma che, anzi, troppo spesso scivola nell'aneddotica. Non era comunque facile destreggiarsi tra l'Ibn al-Mubārak combattente e testimone oculare, e l'autore e trasmettitore onorato dalle più importanti raccolte di hadît, anche se già solo questa molteplicità di ruoli avrebbe costituito motivo di indagine specifica. ${ }^{11}$

\section{Teologia e sufismo}

Tra i volumi della nuova collana di ricerche sull'ibadismo pubblicata da Georg Olms non poteva mancarne uno dedicato in modo specifico alla teologia, curato da Ersilia Francesca. ${ }^{12}$ La Curatice ha premesso un'utile descrizione della

11 Inevitabile, in tale situazione - ma anche per la crescita incontrollabile della bibliografia negli ultimi anni-, che manchi qualcosa nell'elenco finale della bibliografia. In questo caso però alcune assenze sono piuttosto significative, ad esempio Tor, D.G. Violent Order: Religious Warfare, Chivalry, and the Ayyār Phenomenon in the Medieval Islamic World. Würzburg, 2007, p. 53-62; e Denaro, R. "From Marw to the tugiūr: Ibn al-Mubārak and the shaping of a biographical tradition”. Eurasian Studies, 7 (2009), 125-144.

12 Ibadi Theology. Rereading Sources and Scholarly Works. A cura di E. Francesca. "Studies on Ibadism and Oman" n. 4, Hildesheim—Zürich—New York, Georg Olms Verlag, 2015, 331 p., con premessa di Shaykh Abdullah Bin Mohammed Al Salimi, introduzione della Curatrice e contributi di E. Francesca, W. Madelung, R. Al-Sayyid, J. Van Ess, J.C. Wilkinson, S.A. Al-Busaidi, C. Aillet, D. Radivilov, A. Amara, M. Ech-Cheikh, A.M. Di Tolla, L. Chiarelli, A. Al-Salimi, L. Mizopuri e F. Al-Jabiri, M. Dridi, Y. Kondo, D. Leonard, A. Ismail, B. Scarcia 
tradizione italiana e soprattutto napoletana di studi sull'argomento, prima di quattro parti che raccolgono i numerosi contributi su svariati argomenti. Una prima e più breve sezione di ordine storico è seguita da una parte consistente di analisi di fonti. La terza sezione ha per tema il discorso teologico, letto attraverso una serie di studi specifici, in cui la questione delle origini e della prima letteratura ha un ruolo centrale. La quarta e ultima parte è invece dedicata alla teologia contemporanea. Molti, troppi, i capitoli degni di menzione in un volume che ha in ogni caso nello sguardo di insieme il suo punto forte. I contributori, nel loro complesso, ricostruiscono i tasselli di una storia particolare, tutt'altro che marginale, e sempre in dialogo con le tematiche teologiche più significative della storia islamica. L'apertura sul moderno offre poi una chiave di lettura di ciò che agita oggi le comunità musulmane e in particolare quelle minoritarie in una situazione non semplice e con spinte omologanti sempre più forti.

Camilla Adang, Hassan Ansari, Maribel Fierro e Sabine Schmidtke hanno curato un volume dedicato al takfir, ovvero la scomunica o accusa di miscredenza usata nel corso della storia islamica per attaccare avversari. ${ }^{13}$ L'Introduzione traccia le linee fondamentali di un principio utilizzato con una certa frequenza da correnti o gruppi rivali oppure da autorità di vario tipo per contrastare divergenze e opposizioni pericolose, nonostante la diffidenza ufficiale per uno strumento di questo tipo testimoniata dalla stessa tradizione islamica. Questo accadde comunque fin dalla prima generazione islamica e continua fino ad oggi, ovviamente con ripercussioni, atteggiamenti ed effetti diversi per luoghi e tempi diversi. La divisione in due parti riflette la molteplicità di approcci. Nella prima, divisa a sua volta in più sezioni, si percorre il fenomeno dalle origini fino all'età contemporanea tramite i casi specifici trattati dai vari studiosi coinvolti. La seconda parte, in un'unica sezione, analizza varie questioni connesse al takfir, dal punto di vista teologico, filosofico etc. I contributi vanno poi dai primi casi che emergono in ambienti settari del primo islam alle formulazioni e le costruzioni classiche medievali, fino al ritorno del takfir oggi. Particolarmente felice ci pare proprio la scelta di aprirsi all'età

Amoretti, Y.D. Addoun, V.J. Hoffman, A.C. Higgins, A. Ghazal, M. Ouinten, F. Bouchiba, A.R. Coppola.

13 Accusations of Unbelief in Islam. A Diachronic Perspective on Takfir. A cura di C. Adang, H. Ansari, M. Fierro e S. Schmidtke, "Islamic History and Civilization. Studies and Texts" n. 123, Leiden-Boston, Brill, 2016, xviii + 534 p., con introduzione dei Curatori e contributi di E. Francesca, S. Judd, I.T. Krystó-Nagy, D. de Smet, S. Brentjes, A. Levanoni, O. Mir-Kasimov, S. Evstatiev, S. Rizvi, B.J. Didier, J. Nedz, J. Wagemakers, R. Badry, G. Larsson, H. Modarressi, R. Gleave, I.A. Rabb, Z. Szombathy, M. Ebstein. 
contemporanea - periodo in cui l'uso del takfı̈r è particolarmente pronunciato e in casi tra loro lontani (vedi i contributi di B.J. Didier, J. Nedz, J. Wagemakers, R. Badry, e G. Larsson). Senza entrare nel dettaglio dei contributi si può affermare che nel complesso tutti servono allo scopo dettato dal volume stesso, ovvero offrire un ritratto completo ed esaustivo di un aspetto del credo islamico particolarmente significativo e anche discusso nel corso della storia, e ben presente nelle varie componenti dell'islam contemporaneo.

L'edizione commentata della Risālah al-mufidah di 'Alī b. Muhammad b. alWalīd curata da Wilferd Madelung e Toby Mayer è l'occasione per discutere delle allegorie avicenniane connesse all'anima. ${ }^{14}$ L'introduzione di T. Mayer è un vero e proprio saggio che delinea le questioni storiche connesse alla qașidah attribuita a Ibn Sīnā (m. 1037), oggetto del commentario di Ibn al-Walīd (m. 1215), e discute i dubbi avanzati da studiosi precedenti e i riferimenti ad allegorie dello stesso tipo. Il testo ricostruito da Madelung si basa su tre manoscritti conservati presso l'Institute of Ismaili Studies e di cui uno viene usato come base, secondo consuetudini editoriali dei testi proposti nella collana.

La figura e l'opera di al-Ġazālī continuano ad attirare l'attenzione di studiosi. Non potrebbe essere diversamente dato l'importanza del pensiero e dell'opera e la molteplicità di interessi di al-Ǵazālī. Frank Griffel ha curato il secondo volume della raccolta di studi in occasione del $900^{\circ}$ anniversario della sua morte, che include una raccolta di studi che hanno per tema la questione del rapporto tra islam e razionalità alla luce dell'impatto dell'opera gazaliana. ${ }^{15}$ Vale per questo volume quanto scritto nella precedente edizione di questo bollettino per il primo, ovvero che i contributi nel loro complesso analizzano il tentativo di combinare, e le sue ricadute, speculazione razionalistica e islam. ${ }^{16}$ Il quadro che ne emerge anche in questo caso, affrontato

14 Avicenna's Allegory on the Soul. An Ismaili Interpretation. An Arabic Edition and English Translation of 'Al̄̄ b. Muhammad b. al-Walìd's al-Risāla al-mufida. Ediz. testo arabo di W. Madelung, trad. e intr. di T. Mayer. "Ismaili Texts and Translations Series" n. 22, London-New York, I.B. Tauris-The Institute of Ismaili Studies, 2016, xi + 168 + 40 (in ar.) p.

15 Islam and Rationality. The Impact of al-Ghazālì. Papers Collected on His gooth Anniversary. Vol 2. A cura di F. Griffel. "Islamic Philosophy, Theology and Science. Texts and Studies" n. 98, Leiden-Boston, Brill, 2015, xx + 344 p., con introduzione del Curatore e contributi di T. Kukkonen, U. Rudolph, S.R. Ogden, A. El Shamsy, A. Shihadeh, A.A. Akasoy, F. Griffel, D. Janos, J. Janssens, M.S. Ozervarl, M. Riexinger, K. Garden.

16 Il primo volume è apparso l'anno precedente: Islam and Rationality. The Impact of al-Ghazālì. Papers Collected on His gooth Anniversary. A cura di G. Tamer. "Islamic Philosophy, Theology and Science. Texts and Studies" n. 94, Leiden-Boston, Brill, 2015. Vedi su quest'opera “Bollettino di Islamistica”, Oriente Moderno, 96 (2016), 355-356. 
da più punti, è che al-Giazālī risponde a Ibn Sinā attraverso un percorso di razionalizzazione che non può evitare ambiguità nell'adattare i dati scritturali alla sua sintesi. In particolare, tra tutti i contributi, segnaliamo quelli che trattano della sua ricezione in Faḩr al-Dīn al-Rāzì (d. 1209) (D. Janos, J. Janssens) e quelli che guardano all'eredità del percorso e dell'opera di al-Ġazālī nella realtà moderna e contemporanea del mondo islamico (M. Riexinger, K. Garden) e il suo utilizzo nella questione del rapporto con scienze e modernità occidentale — aspetto messo ben in luce anche da Griffel nella sua premessa iniziale.

Alla fruttuosa collaborazione di Wilferd Madelung e Sabine Schmidtke si deve la pubblicazione dei problematici frammenti di due trattati di teologia mu'tazilita attribuiti al visir buyide al-Ṣaḥib Ibn 'Abbād (m. 995) provenienti dalla Geniza. ${ }^{17}$ Si tratta di una figura nota e a cui è dedicato un buon numero di studi, anche se non strettamente connessi alle questioni più direttamente legate alla politica religiosa perseguita, qui percorsa dai due Curatori. I frammenti proposti e tratti dalla collezione Firkovitch di San Pietroburgo non permettono che ipotetiche attribuzioni ad alcune delle opere che gli sono attribuite. Solo la consistenza del primo dei due testi rende plausibile l'identificazione, pur congetturale e non definitiva, con il Kitāb Nahğ al-sabìlfı̀ al-ușūl. I manoscritti sono in caratteri arabi ed ebraici e il loro carattere frammentario rende ancor più significativo lo sforzo operato dai Curatori di ricostruirne ordine e significato, aggiungendo così un ulteriore tassello alla recente attenzione verso $i$ testi mu'taziliti o, in genere, di teologia.

Segnaliamo in questa sezione l'edizione curata da Joep Lameer della traduzione in arabo fatta da Rukn al-Dīn Muḥammad b. 'Alī b. Muḥammad Ğurğānī (XIV sec.) dell'Akhlāq-e Nāṣerī di Nașīr al-Dīn al-Ṭūsī (d. 1274) che è uno dei più celebrati e importanti trattati di etica prodotti nel mondo islamico. ${ }^{18} \mathrm{La}$ parte introduttiva in inglese tratta, come da consuetudine, della vita e dell'opera di Nașīr al-Dīn Ṭūsī, autore assai prolifico e che lasciò opere scritte in arabo e persiano. Il testo in oggetto è un'ampia trattazione dei principi etici ripresi dalle tradizioni greche, persiane, arabe e islamiche in una sintesi di indubbia originalità e notevole valore. L'edizione si basa sull'unico manoscritto noto di questa traduzione, le cui caratteristiche, contenuti e rapporto con l'originale persiano sono discussi in profondità, prima dell'edizione dell'opera condotta in maniera attenta e con numerose annotazioni a piè pagina.

17 Al-Ṣăhib Ibn 'Abbād Promoter of Rational Theology. A cura di W. Madelung e S. Schmidtke. "Islamic History and Civilization. Studies and Texts" n. 132, Leiden-Boston, Brill, 2016, $101 \mathrm{p}$.

18 The Arabic Version of Țüsı̀s Nasirean Ethics. A cura di J. Lameer. "Islamic Philosophy, Theology and Science. Texts and Studies" n. 96, Leiden—Boston, Brill, 2015, ix + 550 p. 


\section{Diritto}

La gestione del dubbio nelle pratiche legali del primo islam in fatto di diritto penale e di applicazione di pene h̆udüd è l'argomento di un corposo e stimolante saggio di Intisar A. Rabb. ${ }^{19}$ L'obiettivo è ovviamente quello di sfidare una visione rigida nell'applicazione del diritto penale di origine coranica e di mostrare, invece, come nell'esercizio storico di tali punizioni le diverse scuole hanno discusso a lungo e con esiti complessi. L'Autrice dimostra con efficacia come è il dubbio metodico che costruisce la discrezionalità che forgia direttamente molte delle peculiarità dell'attività giurisprudenziale nel primo islam e nella definizione dei tratti costitutivi delle scuole giuridiche. Una prima parte del volume, a tal fine, discute gli aspetti tematici connessi all'esercizio del dubbio, quali il rapporto tra una generica severità e un'opposta permissività e poi i riferimenti testuali e interpretativi alla base della legittimità di tale esercizio, insieme alle questioni morali e genericamente sociali sottese a azioni legali nelle due opposte direzioni. Nella seconda parte si analizzano le vie interpretative diverse prese dalle scuole giuridiche sunnite e dalla discussione giuridica sciita imamita evidenziando non solo il procedere tra le due diverse linee ma anche in relazione alla discrezionalità che ciò implica. Nel complesso si tratta di un saggio documentato in maniera puntuale ed efficace, che parte dalle fonti per ricostruirne dinamiche e con piena consapevolezza della retorica e natura polemica dei testi. Ne esce un ritratto che restituisce la complessità dell'elaborazione giuridica dal II/VIII al x/XVI secolo con il giusto equilibrio tra profili tematici e ricostruzione di un'evoluzione storica. Non vi è dubbio, al di là della tematica specifica trattata, che questo saggio debba essere additato ad esempio in quanto esemplifica in maniera ottimale il giusto rapporto tra uso di fonti letterarie e capacità di lettura e di ricostruzione su queste fonti per delineare le peculiarità di una discussione giurisprudenziale ampia e fortemente connessa.

\section{Letteratura islamica}

La storiografia islamica, soprattutto nelle sue parti sulla storia pre-islamica e del primo islam, appartiene pienamente alla letteratura religiosa. Heather N. Keaney ha analizzato le sue peculiarità autoriali saggiando le opere più importanti scritte dal IX al XIV secolo su un tema particolare: la biografia di 'Uțmān

19 Rabb, I.A. Doubt in Islamic Law. A History of Legal Maxims, Interpretations, and Islamic Criminal Law. "Cambridge Studies in Islamic Civilization", Cambridge, Cambridge University Press, 2015, xiii + 414 p. 
b. 'Affān..$^{20}$ La scelta è ovviamente significativa a illustrare diversità di punti di vista e modalità nell'uso delle tradizioni tramandate $(a h b a \bar{a} r)$ con il fine di evidenziare le specificità dei vari autori in base alla citazione o no dei materiali e alla loro interpretazione. Il compito è particolarmente difficile, eppure l'analisi dell'Autrice è sostanzialmente riuscita e importante. Il saggio dimostra come nel valutarne la biografia, storici in condizioni diverse e di orientamenti diversi abbiano affrontato il dilemma del rapporto tra un 'Uțān compagno del Profeta e la sua controversa attività califfale. Realtà storiche diverse, posizioni opposte tra sunniti e sciiti e un mutare nel tempo dell'agenda degli autori determinano l'utilizzo dei materiali e la loro interpretazione, con scelte a volte non necessariamente in linea con posizionamenti o attitudini dei singoli autori. Un aiuto fondamentale nell'intelligibilità dell'analisi deriva dall'organizzazione del volume, che affronta la storiografia diacronicamente e saggiandola però sulle tematiche più significative: il rapporto tra 'Uțmān compagno e califfo; le letture diverse sunnite e sciite sulla šürà che ne determinò l'elezione; il rapporto tra autorità religiosa e politica; e, infine, il giudizio sulla ribellione che ne determinò la morte. Unico punto su cui si deve richiamare l'attenzione, ma che non deve suonare a detrimento di un lavoro di grande interesse, è la definizione di storiografia come genere distinto da quello delle fadă $\overrightarrow{i l}$. Non vi è alcun dubbio che gli interessi che determinano la scrittura di storiografia sono diversi da quelli, correlati alle scienze di hadìt, di coloro che scrissero dizionari biografici e raccolte di fad̆ẳil di compagni del Profeta e di altre figure eminenti. Tuttavia, una distinzione e separazione netta non è sempre facile da tracciare soprattutto per quanto riguarda le singole tradizioni tramandate. Inoltre, laddove vi è differenza, non va sottovalutato il "valore" sociale ridotto attribuito alla scrittura di opere di storiografia, genere letterario quasi sempre considerato di prestigio ridotto rispetto ad altri. Per tale ragione non ci si deve stupire se i presunti modelli di produzione storiografica, come ad esempio al-Ṭabarī, considerato centrale soprattutto in età contemporanea ma non nella storia islamica, non hanno avuto particolare seguito o rilevanza. Questo è però in fondo ciò che rende la storiografia un ambito per certi versi più duttile alle scelte autoriali, e l'Autrice lo dimostra in modo brillante.

Il tema delle concezioni politiche nell'islam ha attratto nel più recente passato molto interesse e ha visto la pubblicazione di numerose opere. Christian Jambet ne tratta prendendo in esame un autore specifico, lo sciita Mullā Șadrā, e la sua concezione politica del mondo quale emerge dal suo sistema

20 Keaney, H.N. Medieval Islamic Historiography. Remembering Rebellion. "Routdlege Research in Medieval Studies” n. 5, London-New York, Routledge, 2013 (pbk 2015), xx + $187 \mathrm{p}$. 
teologico. ${ }^{21}$ Come argomenta l'Autore, si tratta di un pensiero politico quanto mai significativo, perché espressione della sovranità safavide nel suo periodo più importante, alla cui luce viene letta la concezione della sovranità divina, attraverso il confronto con le concezioni teologiche di altri autori musulmani o di altri ambiti religiosi e culturali. La chiave di lettura è giustamente quella mistica e spirituale in un sistema sofisticato che riconosce a questa un valore superiore rispetto alla speculazione di natura giuridico-tradizionale. Il saggio contiene comunque molto altro, e in quasi cinquecento pagine molti sono i temi toccati. Escatologia, implicazioni filosofiche e soprattutto la terminologia utilizzata sono costantemente accompagnate da una rassegna sull'opera di Mullā Ṣadrā nel suo complesso e da puntuali riferimenti nelle varie direzioni. Una parte consistente discute poi di esegesi coranica e del suo approccio filosofico alla questione del commento del Corano (p. 145 sgg.). Segue poi la discussione di argomenti specifici, quali l'esegesi del versetto del Trono (p. 171229), lo hadìt della creazione dei nomi divini (p. 231-259), l'essenza della profezia e la natura profetica (p. 279-369), l'imamato (p. 370-403). Il risultato finale è la descrizione puntuale di un pensiero complesso ma coerente.

La letteratura polemica anti-cristiana costituisce un genere specifico in cui 'Alī al- Țabarī è sicuramente uno dei nomi più antichi e significativi. Un volume curato da Rifaat Ebied e David Thomas presenta edizione e traduzione, dopo un'ampia introduzione, dei suoi al-Radd 'alā al-Nașārà e del Kitāb al-Dìn wa-ldawlah. ${ }^{22}$ Tale lavoro rende giustizia di un Autore come 'Alī b. Rabbān al-Ṭabarī, relativamente poco indagato, e di opere spesso citate e ancor più di frequente utilizzate, anche se finora disponibili in edizione problematiche (nonostante il lavoro pionieristico ed eccellente svolto da Alphonse Mingana che curò un'edizione il Kitāb al-Dìn wa-l-dawlah tra 1922 e 1923). L'introduzione si confronta con i dati sulla sua vita e le sue opere: la sua condizione di cristiano convertito all'islam e la sua fama come medico che operò in Țabaristān e poi presso la corte califfale tra 835 e 860 ca. sono forse le informazioni certe più significative e ripetute da tutte le fonti biografiche. Dopo questa parte introduttiva il volume entra nelle questioni testuali, dando informazioni sui manoscritti utilizzati, tutti quelli conosciuti, per le due opere edite e tradotte, che sono un solo testimone per il Radd e due soli, di cui uno scoperto solo di recente, per il Kitāb al-Dinn. La scarsità di testimoni rende ancor più significativa e problematica la condizione dei testi conservati, in Medio Arabo e con peculiarità

\footnotetext{
21 Jambet, C. Le gouvernement divin. Islam et conception politique du monde. Théologie de Mullā Ṣadrā. Paris, CNRS Éditions, 2016, 470 p.

22 The Polemical Works of 'Alī al-Tabarī. A cura di R. Ebied e D. Thomas. "History of ChristianMuslim Relations" n. 27, Leiden—Boston, Brill, 2016, vii + 491 p.
} 
distintive dall'uso classico consistenti, con tutto ciò che questo implica nelle scelte di edizione e di presentazione del testo ricostruito. ${ }^{23}$ Oltre a ciò i due Curatori evidenziano anche quei costrutti connessi alla conoscenza del siriaco da parte di 'Alī al-Ṭabarī. Il Kitāb al-Dìn wa-l-dawlah è comunque il testo più significativo e importante, essendo, come giustamente si sottolinea, una delle prime opere di daläil al-nubuwwah e quindi una testimonianza particolarmente antica su concezioni e tradizioni circolanti sul Profeta Muhammad e il loro utilizzo in chiave polemica e di dibattito inter-religioso. I dubbi sull'autenticità vengono percorsi e respinti grazie a quelle ricerche più recenti che ne hanno messo in luce attestazioni e utilizzi da parte di autori medievali di diversa estrazione. Segue infine l'eccellente edizione con la traduzione a fronte.

Stesso genere di letteratura polemica è rappresentato dal Radd al-ğamìl attribuito ad Abū Ḥāmid al-Ġazālī (m. 1111), edito e tradotto nella stessa collana per le cure di Mark Beaumont e Maha El Kaisy Friemuth. ${ }^{24}$ Le questioni centrali dell'opera conservata in tre manoscritti sono rappresentate dalla discussione sul suo Autore e le controversie riguardanti l'attribuzione ad al-Ġazālī. Gli argomenti in un senso o nell'altro vengono affrontati offrendo un resoconto utile sullo stato della ricerca e discutendo i numerosi interventi precedenti, ad esempio in merito alle affinità tra i contenuti dell'opera e il pensiero di alĠazāli che rendono plausibile l'ipotesi che l'opera sia originata dalla sua scuola o quanto meno da qualche allievo o autore da lui fortemente influenzato. Alla fine, soppesando tutti gli argomenti, i Curatori propendendo per una non attribuzione ad al-Ġazāli, anche se ciò viene affermato con estrema prudenza. Dopo un inquadramento storico e una breve discussione sul significato dell'opera nell'ambito della letteratura polemica musulmana anti-cristiana, e una elementare descrizione dei manoscritti e dei criteri adottati, segue l'edizione del testo arabo accompagnato dalla traduzione inglese. Si tratta in conclusione di una nuova edizione che riassume lo status questionis sull'opera e che ha molti meriti ma pecca certamente nel dedicare troppo poco spazio alla riflessione critica e testuale mentre invece, nell'analisi dei contenuti, si rivela utilissima.

23 La definizione di Medio Arabo è data dai Curatori e i tratti peculiari sono netti e ben definiti dalla letteratura specialistica in proposito, anche se, va detto, alcuni di questi, riflettono usi assai diffusi anche indipendentemente da altri solitamente definiti Medio Arabo. Ad esempio, la variabilità estrema della grafia della hamza è fenomeno frequentissimo e costante. La storia dell'arabo scritto attestato nei manoscritti nei tratti definiti come classici o appartenenti alla varie forme di Medio Arabo deve ancora essere scritta, anche se il numero di studi disponibile è ormai consistente.

24 Al-Radd al-jamīl. A Fitting Refutation of the Divinity of Jesus Attributed to Abü Hāmid al$\dot{G} a z \bar{a} l \bar{l}$. A cura di M. Beaumont e M. El Kaisy Friemuth, "History of Christian-Muslim Relations" n. 28, Leiden-Boston, Brill, 2016, vii + 207 p. 
Un raccolta di studi su Ǧalāl al-Dīn al-Suyūṭī (d. 1505), il poligrafo islamico per eccellenza, curata da Antonella Ghersetti, non poteva che essere inserita in questa sezione sulla letteratura islamica. ${ }^{25} \mathrm{Il}$ volume raccoglie una serie di saggi su tematiche specifiche che entrano in profondità nelle scelte autoriali di al-Suyūṭi su alcuni precisi argomenti, cercando, in generale, di determinarne peculiarità e originalità nel quadro della realtà dell'Egitto mamelucco della seconda metà del XV secolo. Solo alcuni discutono tematiche più direttamente religiose. É. Geoffroy affronta brevemente la questione della sua difesa del sufismo; A. Spevack ne segue per certi versi le orme percorrendo in maniera asistematica un lavoro dedicato alla sua affiliazione shadhilita e al sufismo in generale;26 J. Kindinger ritorna sulla questione del țaylasān, che Suyūṭi difese contro altre opinioni, inquadrando il dibattito in ambito mamelucco e le questioni sottese nei dibattiti relativi alla liceità di determinati indumenti; S.R. Burge analizza l'Itqān fì 'ulūm al-dìn per evidenziare rielaborazioni e utilizzo di sue e altre fonti precedenti; J. Blecher discute di vari aspetti dei suoi commentari sulle opere di letteratura di haadīt di Buhārī e Muslim. Tutti studi ineccepibili, si è detto, ed attenti a ricostruire realtà storica di produzione delle opere prese in esame in un più ampio contesto. Questo ne costituisce la forza ma anche il limite. Infatti, se da un lato l'insieme ci restituisce per la prima volta i tanti Suyūṭi dei dettagli, interi capitoli della sua produzione, quali l'attività esegetica, la sconfinata produzione di letteratura di hadit su tematiche specifiche o in opere enciclopediche (su tutte il suo Ğāmic) e tutto il resto (basti pensare alle sue raccolte dedicate alle daläil al-nubuwwa) ne restano esclusi. Sarà necessario pensare a un altro volume, di questa qualità, che entri a piene mani nell'agenda di un autore prolifico e complesso come Suyūṭī, per tentare di colmare lo iato tra specificità e l'ampia portata della sua bibliografia.

Le storie e biografie dei medici della tradizione islamica non sono propriamente letteratura di argomento religioso, anche se la letteratura di tabaqāt è fatta di opere generali e di altre specifiche dedicate a determinate professioni. Quella sui medici e tutta la letteratura che discute di condizioni di vita e ruolo nella civiltà islamica secondo le testimonianze letterarie in senso lato è non

25 Al-Suyūțī. A Polymath of the Mamlūk Period. Proceedings of the Themed Day of the First Conference of the School of Mamlūk Studies (Ca' Foscari University, Venice, June 23, 2014). A cura di A. Ghersetti. "Islamic History and Civilization. Studies and Texts" n. 138, LeidenBoston, Brill, 2016, viii + 270 p. con introduzione della Curatrice e contributi di É. Geoffroy, A. Spevack, T. Ito, C. Mauder, M. Banister, C.B. Bahl, S.R. Burge, J. Blecher, F. Grande, J. Hämeen-Anttila, D.R. Filanescu.

26 Con inclusa stereotipata visione caricaturale dell'Orientalismo sugli sviluppi del discorso legale (p. 28), che, si afferma, sarebbe giunto, secondo la lettura orientalistica, a compimento e chiusura nel IX sec. (?). 
meno significativa ed è nel ricco saggio di Johann Sebastian Bürgel passata in rassegna ed analizzata in maniera esaustiva, secondo antiche consuetudini di acribia e di metodica letture dei materiali, e senza ricorso a teorizzazioni di varia provenienza. ${ }^{27}$

\section{Storia dell'islam}

Le possibilità offerte dai nuovi strumenti digitali anche per la ricerca storica sono ben esemplificate dal ponderoso saggio che Amikam Elad ha dedicato alla storia della rivolta di Muhammad al-Nafs al-Zakiyyah (m. 762). ${ }^{28}$ L'Autore ricostruisce vicenda biografica, partecipazione alla rivolta anti-'abbaside ed esiti passando in rassegna una mole notevole di dati, alla ricerca di coerenza, attendibilità e plausibilità storica analizzando contenuti e trasmettitori coinvolti. L'ordine seguito è teso a ricostruire il quadro storico, in primo luogo attraverso le notizie biografiche sulla collocazione di Muhammad b. 'Abd Allāh nell'ambito sciita o proto-sciita e nel ramo hasanita in particolare. Dopo di ciò si discute nel dettaglio il confronto con il califfo al-Manșūr generato dalla rivolta del 762 che portò alla breve occupazione di Medina, presto sbaragliata dall'esercito inviato per sedarla e alla sconfitta dei rivoltosi. La ricostruzione, ad esempio, dei combattimenti, cerca di districarsi con i dati, numerosi, da cui però non è agevole stabilire con certezza la cronologia. L'Autore lo esplicita subito e tenta quindi di "mettere in fila" le innumerevoli tradizioni. La seconda parte del saggio è una rassegna esaustiva degli schieramenti e quindi chi era favorevole e chi no alla ribellione, passando in rassegna lo schieramento qurayshita, quello 'alide e poi le posizioni tribali arabe. Interessante è il capitolo che discute delle prese di posizioni di esperti di ḩadīt e di faqīh, tra cui, ad esempio, Mālik b. Anas. Abbiamo così un ritratto minuzioso e completo a cui si può solo aggiungere una considerazione finale, ovvero se da un quadro così dettagliato non possa venire a mancare un necessario sguardo di insieme. La ricchezza dei dati e le questioni affrontate rischiano di produrre un ritratto biografico-genealogico che ha il merito di correggere tanti dettagli senza però produrre una visione complessiva necessaria.

27 Bürgel, J.S. Ärztliches Leben und Denken im arabischen Mittelalter. A cura di F. Käs, "Islamic History and Civilization. Studies and Texts" n. 135, Leiden-Boston, Brill, 2016, xxxvi + $534 \mathrm{p}$.

28 Elad, A. The Rebellion of Muhammad al-Nafs al-Zakiyya in 145/762. Tālibīs and Early Abb$\bar{a} s \bar{s}$ in Conflict. "Islamic History and Civilization. Studies and Texts" n. 118, LeidenBoston, Brill, 2016, xi $+527 \mathrm{p}$. 
Una raccolta di saggi sul contributo dei non musulmani, soprattutto cristiani, nello stato islamico umayyade, curata da Antoine Borrut e Fred M. Donner, inaugura una nuova serie dell'Oriental Institute dell'Università di Chicago. ${ }^{29} \mathrm{La}$ raccolta evidenzia un aspetto più volte sottolineato da queste pagine anche in merito allo sviluppo delle concezioni religiose, ovvero il fatto che i musulmani furono per lungo tempo una minoranza anche esigua nei territori conquistati e che, di conseguenza, il contributo dei non musulmani alla costruzione dell'Impero islamico fu fondamentale e continua nei primi secoli. Il volume, nel suo insieme, evidenzia complessità e assenza di confini netti di qualsiasi tipo: sociali, linguistici e anche, seguendo le teorie di Donner sui mu'minūn/ muslimūn, religiosi. L'influenza e il ruolo dei non musulmani è evidente, soprattutto da parte dei cristiani che costituivano la maggioranza della popolazione siriana, rispetto alle altre confessioni presenti, ad esempio ebrei e zoroastriani. Tra tutti i capitoli segnaliamo in particolare quello di S.H. Griffith su Giovanni Damasceno, che discute le altalenanti testimonianze sul suo impiego presso la corte califfale; quello più esteso di W. al-Qāḍī, che analizza i dati contenuti nelle fonti arabe e non arabe sulla partecipazione dei non musulmani nell'esercito musulmano, tracciando alcune considerazioni significative e inedite, anche sulle possibilità di utilizzo di queste testimonianze letterarie in senso lato; e infine quello di S. Pinckney Stetkevych, che evidenzia, ancora una volta, l'utilità unica della poesia per comprendere la regalità umayyade, così come già fatto da P. Crone e M. Hinds in un loro saggio famoso ma, ancor prima di loro, V. Strika in un misconosciuto articolo. ${ }^{30}$

La figura e l'opera di Patricia Crone sono state una costante di queste pagine. A breve distanza dalla raccolta di studi in suo onore e dalla sua scomparsa (2015) sono stati raccolti in tre volumi i suoi contributi sparsi in articoli. ${ }^{31}$ In

29 Christians and Others in the Umayyad State. A cura di A. Borrut e F.M. Donner, "Late Antique and Medieval Islamic Near East", Chicago, The Oriental Institute of the University of Chicago, 2016, ix + 213, con introduzione dei Curatori e contributi di D. Whitcomb, S.H. Griffith, M. Debié, T. Daryaee, W. al-Qāḍī, S. Pinckney Stetkevych, M. Levy-Rubin, L. Yarbrough.

30 Crone, P. e Hinds, M. God's Caliph. Religious Authority in the First Centuries of Islam. Cambridge 1986; V. Strika, "La formazione dell'iconografia del califfo nell'arte ommiade", in Annali dell'Istituto Universitario Orientale di Napoli, 14 (1964), pp. 728-51.

31 Crone, P. The Qur'ānic Pagans and Related Matters. Collected Studies in Three Volumes. A cura di Hanna Siurua, "Islamic History and Civilization. Studies and Texts" n. 129, Leiden-Boston, Brill, 2016, xviii + 503 p.; Crone, P. The Iranian Reception of Islam: The Non-Traditionalist Strands. Collected Studies in Three Volumes. A cura di Hanna Siurua, "Islamic History and Civilization. Studies and Texts" n. 130, Leiden-Boston, Brill, 2016, xiii +368 p.; Crone, P. Islam, the Ancient Near East and Varieties of Godlessness. Collected 
questo caso, più che di un volume simile a quelli della serie Variorum, si tratta di una riproposizione in distinte opere tematicamente omogenee di articoli che hanno una coerenza riconoscibile, scelti dall'Autrice stessa che ha così consegnato ai curatori una sorta di ultima opera che include anche alcuni capitoli inediti. Molti gli spunti sia nel contenuto dei capitoli, già noti, sia in ciò che è aggiunto. Tutti i volumi sono accompagnati da introduzioni dell'Autrice (più breve nel secondo volume). Quella al primo volume è in particolare una sorta di profilo autobiografico del proprio percorso intellettuale attraverso le monografie prodotte e il capitolo finale riproduce il discorso letto in occasione della laurea honoris causa ottenuta nel 2013 a Leida che include una riflessione sui cambiamenti testimoniati dalla Crone nel campo della storia islamica. Nel complesso l'opera evidenzia l'interesse per ogni aspetto storico e religioso sulle origini dell'islam e un'originalità unica sia nel percorrere vie poco battute da precedenti ricerche sia nel tentare nuove chiavi interpretative con una verve polemica e una forza ed energia che sono state proverbiali. Il primo volume, in particolare, mostra al meglio possibilità di indagine nel $\mathrm{e}$ intorno al Corano, mostrando quindi, in maniera forse inaspettata, considerando gli esordi della Crone, la centralità dell'approccio ai testi e cosa se ne può ricavare, per quanto innovativo sia e lontano dalla lettura comune. Nel caso della storia religiosa sulle origini dell'islam, il primo volume mostra in maniera eccellente come non solo le vicende del nascente islam possono essere indagate nel testo sacro, ma anche la complessa e intricata religiosità circostante può essere compresa attraverso un testo problematico ed elusivo come il Corano. Il secondo volume accompagna i temi affrontati anche nella sua ultima monografia, ${ }^{32}$ ovvero guardando all'Iran e alle reazioni religiose che la conquista musulmana suscitò. Nel terzo e ultimo volume l'orizzonte è il Vicino oriente e la situazione religiosa a partire dai dati letterari islamici nelle diverse direzioni. Per la coerenza sostanziale, gli inediti presentati e le rare testimonianze della stessa Crone, i volumi paiono tre raccolte originali, estremamente coerenti e con il marchio preciso di un'autorialità che li toglie completamente dal genere delle miscellanee postume riproposte senza alcuna mediazione.

La storia della costruzione e vera e propria prima definizione dei luoghi di culto islamici all'indomani delle conquiste ha non solo un significato per la storia artistica e dell'architettura dei primi tempi, ma anche per le vicende religiose. Mattia Guidetti analizza questa fase iniziale e il rapporto tra prime

Studies in Three Volumes. A cura di Hanna Siurua, "Islamic History and Civilization. Studies and Texts" n. 131, Leiden-Boston, Brill, 2016, xvii + 261 p. 
costruzioni e adattamento di luoghi di culto musulmani in un ambiente a lungo a maggioranza cristiana come la regione siriana in senso alto. ${ }^{33}$ La questione è centrale anche per ogni aspetto che riguarda la nascente religione islamica, ovvero l'essersi definita nelle realtà di conquista dove le comunità musulmane erano piccole minoranze immerse in maggioranze con cui si dovettero per forza confrontare e definire. Paradigmi interpretativi, riletti intorno al concetto di tarda antichità che tanto successo ha avuto negli ultimi anni e continua ad avere, vengono percorsi attraverso una rassegna della recente storiografia sul primo islam, su cui innestare la lettura critica in ambito storico-artistico, in cui, come ricorda l'Autore, la dinamica tra continuità e rottura è stata meno significativa e dove quindi gli elementi di un periodo tardo-antico che va oltre la novità delle conquiste è una chiave di lettura consolidata. Nel concreto del tema analizzato la questione tocca il rapporto tra chiese e prime moschee e quindi come sia cambiato nella regione siriana il panorama dei luoghi di culto con il sovrapporsi lento ma inesorabile della presenza musulmana a partire dal viI secolo. Si discute quindi della trasformazione di chiese in moschee, della contiguità tra i diversi luoghi di culto e di questioni connesse, fino all'interessante discussione del riutilizzo di materiali, in particolare colonne, per la costruzione di moschee. Il ritratto che ne risulta è avvincente e mostra in maniera efficace le complessità di convivenza e di progressiva costruzione dell'immaginario musulmano di costruzione di spazi sacri. Il saggio è quindi interessante e ricco di suggestioni, e per certi versi coraggioso nel tentativo di tentare una ricostruzione tra storiografia e storia dei luoghi di culto, cercando così di superare quelli che sono spesso comparti separati negli studi. Riuscire pienamente in tale proposito è però compito arduo, perché richiede piena capacità di destreggiarsi tra produzione letteraria ed evidenza archeologica o di fonti primarie di vario tipo, e attraversando filologie diverse. Inevitabilmente ciò che colpisce chi soprattutto si occupa di testi, di retorica dei testi e di problematiche connesse è l'uso ridotto nelle argomentazioni offerte di fonti primarie islamiche che, quando citate, lo sono in edizioni datate con problematiche testuali non indifferenti. ${ }^{34}$

33 Guidetti, M. In the Shadow of the Mosque. The Building of Mosques in Early Medieval Syria. "Arts and Archeology of the Islamic World" n. 8, Leiden-Boston, Brill, 2016, xii + 211 p. + 13 tavole a colori a chiusura del volume.

34 La diffidenza nei confronti della letteratura islamica, storiografia o di ambito geografico che sia, è questione seria e anche condivisibile, ma è questione ineludibile trattando di primo islam, pace l'onda lunga di posizioni critiche esse stesse oggetto di revisione. Il rischio, infatti, è quello di offrire una ricostruzione critica efficace e complessa usando però fonti in maniera acritica e senza consapevolezza dei problemi che queste presentano, 
All'Iran ilhkanide è dedicata la raccolta di saggi curata da Bruno De Nicola e Charles Melville. ${ }^{35}$ La questione centrale è l'impatto dell'invasione mongola tra XIII e XIV secolo nell'ampia regione iraniana includendo anche Anatolia orientale e Azerbaijan. In un volume, perciò, più di storia, i saggi dedicati a questioni religiose sono rispettivamente quello di F. Hodous, che ha per tema la pena di morte nel periodo ilkhanide alla luce dei diversi credo religiosi che ne influenzarono implementazione a fasi alterne, e quello di G. Lane, sulla cosiddetta "Phoenix mosque" di Hangzhou e il suo ruolo di connessione tra Asia Centrale, regione iraniana e Cina.

La storia della città islamica e di conseguenza la costruzione dello spazio urbano delle più importanti città del mondo islamico medievale è invece affrontata da Fuzuko Amabe in un saggio innovativo e stimolante. ${ }^{36}$ Il soggetto è propriamente l'autonomia di alcune città musulmane, che si vuole dimostrare per correggere la visione tradizionale che attribuisce tale autonomia solo alle realtà urbane europee nel corso del Medioevo. Per fare ciò l'Autore ha setacciato nella letteratura araba musulmana ogni possibile accenno che indicasse, in modo più o meno esplicito, un elemento di "autonomia urbana" nelle città prese in esame. I casi studiati sono conosciuti come quelli che hanno vissuto condizioni simili nel corso del Medioevo e dopo la fine dell'unità politica centrale dal IX-X sec., anche se specificità e soprattutto condizioni sociali diverse (anche solo nel rapporto tra élite e classi intermedie e subalterne) nella società islamica rendono i paragoni con la realtà europea non sempre efficaci. Le aree prese in esame, la Siria (Damasco, Aleppo) e al-Andalus (Cordova, Toledo, Valencia), con la sola aggiunta di Tunisi (oltre ai casi falliti della Baghdad del IX-XI secc.), sono i casi più interessanti tra X e XII secolo e sono anche quelli su cui precedenti studi hanno elaborato analisi in tale direzione. Tale studio, alla fine, aggiunge ben poco, articolando la sua analisi su un numero non troppo esteso di fonti primarie e ricercando nelle diverse definizioni di categorie

con rischi non indifferenti, vedi ad es. p. 24 n. 57 dove si cita al-Wāqidī affermando che sarebbe noto attraverso quanto citato da autori successivi senza chiarire di quali opere si sta parlando o se si possa far riferimento alle sue Maghāzz- - pubblicate-o alle svariate opere apocrife a lui attribuite.

35 The Mongols' Middle East. Continuity and Transformation in Ilkhanid Iran. "Islamic History and Civilization. Studies and Texts" n. 127, Leiden—Boston, Brill, 2016, xiii + 346 p., con introduzione dei Curatori e contributi di T. May, R. Amitai, E. Ravalde, B. De Nicola, F. Hodous, M. Biran, J. Kolbas, K. Rührdanz, A. Khanbaghi, B. Dashdondog, G. Lane, B. James, C. Melville.

36 Amabe, S. Urban Autonomy in Medieval Islam. Damascus, Aleppo, Cordoba, Toledo, Valencia and Tunis. "Islamic History and Civilization. Studies and Texts" n. 128, LeidenBoston, Brill, 2016, $\mathrm{x}+225$. 
sociali attestate quell'impegno dellélite che possa suggerire una autonomia di fatto assimilabile ai fenomeni analoghi europei del basso Medioevo. In tutto ciò, evidentemente, il fattore religioso è del tutto assente ed, anzi, la realtà urbana delle città islamiche medievali evidenzia più che altrove il carattere confessionali di molte realtà sociali e storiche.

\section{Islam in aree regionali specifiche}

L'Islam indonesiano nella realtà, soprattutto politica, contemporanea è al centro della minuziosa analisi di Carool Kersten. ${ }^{37}$ L'Autore fotografa il ruolo dell'Islam negli ultimi decenni e nei suoi sviluppi più recenti, discutendo nel dettaglio posizionamenti e ruolo delle diverse organizzazioni, associazioni e formazioni di tipo anche politico sulla scena indonesiana. Se da un lato non si tralascia la funzione storica di Mahdatul Ulama e il ruolo dell'islam negli anni finali di Suharto che vide finire la sua trentennale presidenza nel 1998, lo studio attraversa i fermenti e i problemi soprattutto degli ultimi quindici anni con lo sviluppo democratico più recente accompagnato da un ruolo sempre più significativo di adesione o partecipazione islamica visibile. Tutto ciò è stato caratterizzato da una crescita intricata di attori in gioco e con una interessante dinamica che vede varie forme di islam, associazionismo di vario tipo e musulmani che si sono formati presso centri universitari come la McGill University di Montreal, tutte partecipare e anche contrastarsi sul terreno della legittimità. Il volume, dopo aver tracciato il necessario profilo introduttivo sulle forze intellettuali islamiche all'opera sulla scena indonesiana, affronta alcune delle tematiche di natura politica più sensibili nella nuova realtà indonesiana, quali la questione del secolarismo unita a quelle del pluralismo e del liberalismo; la rilettura della tradizione storica e la legittimazione degli attori in gioco nell'agone politico e sociale; il dibattito sul secolarismo in rapporto alla questione della democrazia, ovviamente sullo sfondo delle novità più recente del dibattito politico indonesiano; lo scontro tra atteggiamenti formalisti e interpretazioni contenutistiche nella questione dell'applicazione del diritto islamico; e, infine, il rapporto tra pluralismo religioso, diritti umani e libertà di pensiero in realtà a maggioranza musulmana. Il lettore vi ritrova, declinati nella realtà dell'Indonesia, molte delle tematiche che attraversano molti paesi musulmani. I processi di re-islamizzazione o di nuova centralità politica e sociale dell'identità o appartenenza islamica comportano un sempre maggior peso del fattore

37 Kersten, C. Islam in Indonesia. The Contest for Society, Ideas and Values. London, C. Hurst \& Co., 2015, $\mathrm{xx}+373 \mathrm{p}$. 
religioso nella realtà sociale e politica. E ciò avviene anche in Indonesia, con esiti significativi nel dibattito sul rapporto tra Islam e alcuni prodotti e concetti della modernità occidentale (secolarismo, liberalismo, diritti umani, libertà di pensiero etc.).

All'Indonesia e ad Aceh guarda anche la raccolta di studi curata da R. Michael Feener, David Kloos e Annemarie Samuels. ${ }^{38}$ I contributi prendono in esame la realtà contemporanea e in particolare gli ultimi dieci anni, anni particolarmente sensibili qui come altrove, per i dibattiti sul ruolo dell'islam in rapporto agli stati e alle diverse condizioni politiche, il tutto definito intorno all'introduzione ufficiale e implementazione della šarîah. Questo in particolare è avvenuto in una realtà complessa e come risultato finale di un processo tutt'altro che lineare e semplice, che ha inevitabilmente condizionato ogni sviluppo successivo per tutto questo breve scorcio di XXI secolo. In tale situazione e in particolare nel contesto conflittuale di Aceh, un ruolo fondamentale di ricomposizione e ridefinizione degli schieramenti ha avuto lo Tsunami del 24 dicembre 2004 e i tragici eventi che l'hanno accompagnato (vedi su questo il contributo di A. Samuels). Gli altri capitoli discutono dei vari ambiti in cui si esercita questa crescente attenzione verso l'implementazione della šaríah in Aceh, ad esempio dal punto di vista etico (D. Kloos); oppure nelle questioni legali islamiche in contrasto con l'attivismo pro-diritti delle donne oppure con l'azione di ONG locali (K. Großmann, D. Afrianty). La questione femminile ritorna anche nel contributo di E. Srimulyani, mentre R. Idria affronta il tema dei punk musulmani e la legge islamica. Chiudono capitoli più generali dedicati alla scena sociale e alle reazioni di gruppi sociali al processo di progressiva "islamizzazione" (B. Otto e J.M. Otto), compresi anche gli acehnesi emigrati (A. Missbach). Proprio questa attenzione alla ricezione "dal basso", e non solo della discussione intellettuale, è forse la caratteristica più significativa di un volume che ha una profonda coerenza e unità di intenti.

A una realtà regionale specifica contemporanea guarda anche lo studio che Rajeswary Ampalavanar Brown ha dedicato all'islam in Tailandia. ${ }^{39}$ Si tratta di un'analisi della condizione minoritaria dei musulmani in una realtà come quella tailandese, caratterizzata da grande varietà nelle stesse comunità, con

38 Islam and the Limits of the State. Reconfigurations of Practice, Community, and Authority in Contemporary Aceh. A cura di R.M. Feener, D. Kloos e A. Samuels. "Leiden Studies in Islam and Society" n. 3, Leiden-Boston, Brill, 2016, xx + 249, con prefazione (dei Curatori) e contributi di R.M. Feener, A. Samuels, D. Kloos, K. Großmann, D. Afrianty, E. Srimulyani, R, Idria, B. Otto e J.M. Otto, A. Missbach.

39 Brown, R.A. Islam in Modern Thailand. Faith, Philantropy and Politics. "Routledge Contemporary Southeast Asia Series” n. 62, London-New York, 2014, xvi + 277 p. 
origini diverse tra i musulmani che vivono nel nord, nel centro e nel sud del paese. Ciò corrisponde anche ad attitudini diverse, tra un islam più vicino alla sensibilità storica delle confraternite mistiche e quello invece, tra i tailandesi Malay, sensibile a richiami più politici. Molti i temi specifici trattati. La diversità storica e sociale è vista attraverso la struttura economica soprattutto delle fondazioni pie (waqf) fino alla realtà contemporanea. Nell'analisi si guarda fino al XIX secolo, così come nel capitolo dedicato a cosmopolitismo e pluralismo legale. Altri capitoli discutono infine delle strutture di educazione islamica, delle questioni sociali connesse al governo della maggioranza Thai, la funzione del pellegrinaggio per le dinamiche interne e la presenza saudita nella più turbolenta realtà della Tailandia. Sullo sfondo di tutta la vicenda e per le questioni trattate ritorna inevitabilmente la problematica del rapporto tra adat e šarīah, e soprattutto il rapporto tra élite musulmane e autorità politiche buddiste. L'Autore mette ben in luce non solo la complessità del rapporto tra componenti diverse e partecipazione e cooptazione più recenti, ma anche come ciò ha determinato politiche di inclusione e di consolidamento della presenza e visibilità politica dei musulmani.

L'Asia meridionale è altro luogo di notevole interesse per conoscere le evoluzioni dell'islam contemporaneo. Michel Boivin e Rémy Delage hanno in particolare indagato negli aspetti devozionali collegati alle pratiche di culto presso santuari e quelle connesse alla mobilità di pellegrini e di viaggiatori. ${ }^{40} \mathrm{Si}$ tratta di una raccolta di undici saggi ricchi di informazioni fin dal capitolo introduttivo di M. Boivin che specifica l'uso del termine "devotional" sia nel quadro degli studi storico-religiosi o islamistici sia in quello dell'analisi della mobilità che vede protagonisti appartenenti a confraternite mistiche e gruppi di musulmani attraverso pratiche di devozione connesse a santuari e luoghi di culto. In tale quadro, particolare attenzione è data alla descrizione di singole figure di "santi" (U. Falasch, D. Ortis) oppure all'analisi di specifici santuari (M. Mubeen, J. Schaflechner) e di pratiche di pellegrinaggio (M. Valdinoci, A. Philippon). La piena consapevolezza dei più recenti approcci, anche disciplinari, degli studi su pratiche devozionali e di pellegrinaggio, è ben presente, così come non sfuggono le complesse questioni che toccano il rapporto tra il cosiddetto islam "popolare" e le diverse forme dell'islam sunnita, sciita e sufi, con la funzione di ricostruire un quadro complesso che ha molteplici utilità. La prima è

40 Devotional Islam in Contemporary South Asia. Shrines, Journeys and Wanderers. A cura di M. Boivin e R. Delage. "Routledge Contemporary South Asia Series", London-New York, Routledge, 2015, Xv + 195 p., con introduzione di M. Boivin e contributi di A. Papas, M. Membrado, O. Kasmani, U. Falasch, D. Ortis, M. Viitamåki, M. Mubeen, J. Schaflechner, M. Valdinoci, A. Philippon. 
innanzitutto quella di analizzare casi specifici in un quadro omogeneo che sa offrire un quadro unitario; la seconda è quella di dare un'ulteriore testimonianza della ricchezza e complessità dell'islam indiano e dell'Asia meridionale più ampia, che riflette un'eredità storica di notevole importanza, ma anche il peso demografico dei musulmani che vi vivono nella realtà mondiale. In forza di ciò, l'analisi degli aspetti devozionali di matrice popolare, in una realtà segnata dai processi di ri-tradizionalizzazione, ha un significato particolare e quanto mai attuale.

All'India musulmana rimanda l'interessante studio che Eric Lewis Beverly ha dedicato allo stato di Hyderabad tra il 1850 e il 1950 circa.$^{41}$ La tesi di fondo è che la sua indipendenza, almeno formale, così come quella di altre simili entità, all'interno del Raj britannico, fu tutt'altro che formale e servì invece a creare zone di mediazione tra il controllo coloniale britannico e spinte di diverso genere che attraversarono soprattutto la comunità musulmana indiana. L'indipendenza di Hyderabad servì, questa la tesi formale, a definire e creare forme di coordinamento e organizzative di musulmani e non necessariamente in connessione diretta con la presenza coloniale europea. Le connessioni con il mondo musulmano al di fuori dell'India lo dimostrano pienamente, anche se in molti casi, aggiungiamo noi, questo è un fattore ancora da studiare a fondo, soprattutto per evidenziare il ruolo decisivo che l'India ha avuto per le spinte riformistiche e per molte novità nel pensiero islamico del XIX secolo. Se questo possa corrispondere a un fattore decisivo per le vie alla modernità del mondo islamico nel suo complesso, è difficile dire e pare un po' ottimistico. É tuttavia indubbio che analisi di questo tipo restituiscono la realtà di parte del mondo islamico o comunque popolato da musulmani, come l'Asia meridionale, ben più articolata della semplicistica visione schiacciata sulla presenza coloniale. Del resto non fu solo Hyderabad a godere di questa relativa indipendenza in cui il carattere islamico della sovranità, anche se parziale e ufficiosamente controllata, ebbe un ruolo importante e determinò anche la necessità di definirne la natura religiosa e il suo ruolo nella nuova realtà storica. In questa linea vengono quindi analizzate le tre aree tematiche scelte e che danno il nome alle sezioni: idee, istituzioni e spazio urbano. Nella prima ritroviamo le parti più significative, dato che si affrontano sforzi riformistici e contatti e confronti con altre realtà musulmane, soprattutto a partire dal 1880. Tutto ciò tocca anche questioni relative alle istituzioni, anche se qui, come nella parte dedicata allo spazio urbano, si prende in esame piuttosto la gestione politica del rapporto

41 Beverly, E.L. Hyderabad, British India, and the World. Muslim Networks and Minor Sovereignty. Cambridge, Cambridge University Press, 2015, xvii + 344 p. 
con la realtà circostante e le questioni di ordine pubblico e di organizzazione della citta di Hyderabad.

Tra gli studi più recenti dedicati all'islam in Russia, quello di Mustafa Tuna analizza le questioni più politiche di rapporti e anche definizione tra stato zarista e comunità soprattutto dell'ampia regione del Volga e degli Urali. ${ }^{42}$ Non si affrontano, di conseguenza, le dinamiche interne nel corso di un secolo, il XIX, decisamente importante e ricco di novità e cambiamenti, bensì le politiche centrali e la questione dell'impatto dei processi di modernizzazione nazionale sulle comunità musulmane, soprattutto per quanto riguarda rapporti e costruzione di processi educativi e organizzativi. Gli interlocutori sono da un lato i promotori di riforme e di definizione stessa dell'identità nazionale russa, e dall'altro il posizionamento delle comunità musulmane locali e delle loro leadership, più da un punto di vista politico che religioso. Gli anni fondamentali, in ciò, sono quelli intorno al 1860 quando da parte russa cominciano ad emergere disagi e dibattiti intorno all'organizzazione scolastica ed educativa e in rapporto alla situazione nelle comunità musulmane che determinano una centralizzazione e un nuovo controllo. Tutto ciò generò contrasti e proteste, con reazioni diverse da parte dei diversi strati sociali delle comunità musulmane davanti alle spinte di "normalizzazione" del governo centrale. Il risultato finale, da una parte e dall'altra, fu il lungo tragitto di trasformazione, nel solco della modernità europea, percorso fino alle soglie del xx secolo e i notevoli cambiamenti sociali e cultuali che hanno investito le comunità musulmane della regione.

Alla storia dellislam in Russia o comunque connesso alle regioni russe si riallacciano anche le vicende della minoranza musulmana tatara del Baltico, oggetto degli studi raccolti da Ingvar Svanberg e David Westerlund. ${ }^{43}$ Le tre parti in cui sono divisi gli otto contributi, inclusa l'introduzione, ricostruiscono le varie ondate (la seconda e terza parte si intitolano infatti "second" e third wave") di arrivo dei tatari, con i primi passi storici e poi un'analisi della regioni raggiunte in tempi diversi, toccando anche, nella terza e ultima parte, Svezia e Germania. Gli aspetti storici e di ricostruzione di percorsi assai diversi, oltre

42 Tuna, M. Imperial Russia's Muslims. Islam, Empire, and European Modernity, 1788-1914. "Critical Perspectives on Empire", Cambridge, Cambridge University Press, 2015, xiii + $276 \mathrm{p}$.

43 Muslim Tatar Minorities in the Baltic Sea Region. A cura di I. Svanberg e D. Westerlund. "Muslim Minorities" n. 20, Leiden-Boston, Brill, 2015, xi + 183 p. con prefazione dei Curatori, introduzione di S. Cwiklinski, e contributi di T. Bairašauskaité e E. Račius, A.S. Nalborczyk, R. Bekkin e S. Stålberg, H. Halén, T. Martikainen, T. Abiline e R. Ringvee, V. Ščerbinskis, S. Stålberg e I. Svanberg, S. Cwiklinski. 
che delle ambiguità stesse della storia dei tatari, procedono insieme alla definizione degli aspetti di fede musulmana che diventa visibile nelle varie realtà pur in condizioni politiche sovente problematiche. Percorso storico e analisi del portato di fede con osservazione etnografica, anche attraverso il ricco apparato di immagini, procedono insieme senza escludersi l'un l'altra, ad evidenziare radicamento e complessità della realtà di queste minoranze islamiche. Per certi versi i capitoli sui singoli paesi diventano quasi ampie voci enciclopediche che coprono ogni aspetto della presenza tatara in una determinata nazione. È del resto la diversità e la mutevolezza delle condizioni e le vicissitudini di questi musulmani europei la chiave di analisi di un percorso che arriva fino alla diaspora contemporanea e alle problematiche del presente nei vari paesi. Il volume nel suo complesso riesce a combinare le diverse esigenze prodotte dalle tematiche differenti, con una sostanziale schematica omogeneità.

Nella storia dell'islam in America il capitolo forse più antico e problematico dal punto di vista storiografico è rappresentato dalla presenza morisca a seguito dei conquistatori spagnoli nel XVI e prima parte del XVII secolo. Karoline P. Cook propone una ricostruzione di questa storia, utilizzando soprattutto materiali di archivio e alcuni casi specifici desunti da documentazione di casi giudiziali. ${ }^{44}$ Lo studio si confronta con le problematiche delle fonti e il carattere elusivo di gran parte della testimonianza storica, in cui l'accusa di essere morisco o musulmano ha una precisa funzione nella costruzione dell'identità imperiale spagnola, in uno scontro che si gioca tutto sui provvedimenti legislativi e la pratica assai diversa che vide musulmani e Moriscos attraversare l'Atlantico e contribuire alla colonizzazione delle Americhe. Ben poco degli aspetti più religiosi viene affrontato nel volume, anche in quei capitoli che si occupano di pratiche magiche e forme di tradizione popolare per cui i Moriscos erano particolarmente rinomati e anche ricercati. Le testimonianze storiche di varia provenienza nelle colonie spagnole ne attestano la continua e significativa presenza, al di là delle restrizioni legislative e quindi evidenziano il contributo musulmano nella costruzione delle nuove realtà americane nate dalla colonizzazione spagnola. Il saggio è sicuramente meritevole e significativo nella ricostruzione che offre. I limiti che evidenzia sono quelli delle fonti a disposizione, che neppure un confronto di metodologie o di discussioni teoriche è in grado di superare.

Le vicende della penisola iberica tra fine della presenza musulmana e inizio dell'età moderna sono complesse e di grandi importanza per la storia della regione, dell'islam in Europa e per gli studi moderni europei su arabo e Islam. 
Una raccolta di studi per le cure di una delle massime studiose in tale ambito, Mercedes García-Arenal, discute un tema specifico, ovvero le dinamiche di conversione al cristianesimo di ebrei e musulmani innestate da Reconquista ed espulsioni. ${ }^{45}$ L'introduzione già evidenzia tutti i temi connessi, dalla questione delle relazioni culturali e il passaggio di nozioni, anche attraverso pratiche di traduzione, alle polemiche su nobiltà di sangue, dissimulazione e commistioni consce o inconsce di una scena culturale ricca e complessa come quella spagnola. Le tre parti del volume sono dedicate a ebrei e alla circolazione della Bibbia, alla conoscenza di Corano e diffusione di conoscenza dell'islam a partire dalla penisola iberica e, infine, allo spazio indefinito e significativo di ipocrisia, conversioni non sincere e l'uso polemico di tali categorie. I casi specifici analizzati da ognuno sono in genere di eccellente valore informativo. Per quanto riguarda in particolare la sezione e le parti dedicate all'islam segnaliamo il contributo di P.M. Tommasino che analizza una questione specifica come la resa di 'alaq nella sura 96 tra da traduzioni latine e polemica religiosa alimentate da conoscenze limitate in circolazione nell'Europa dell'età moderna; quelli di R. Szpiech, T. Soto e K.K. Starczewska, dedicati a Juan Andrés e con tagli piuttosto diversi e che insieme contribuiscono a delinearne ulteriormente figura e circolo culturale di appartenenza (tra cui spiccava Joan Martín de Figuerola); l'impatto della polemica inter-religiosa nella circolazione di conoscenze sull'islam in Nordeuropa, avvenuta in genere attraverso la mediazione di Moriscos (G. Wiegers); e le dinamiche relativa ai convertiti di ascendenza islamica e le problematiche connesse nella Spagna cattolica (M. García-Arenal).

\section{Islam moderno}

Sebbene sotto questa titolazione sono di solito trattati aspetti dell'Islam del XIX secolo, l'importante saggio che Khaled El-Rouayheb ha dedicato alla storia intellettuale del XVII ci induce a allargare tale ambito. ${ }^{46}$ Il saggio del resto dimostra come tale periodo fu tutt'altro che segnato da stagnazione/

45 After Conversion. Iberia and the Emergence of Modernity. A cura di M. García-Arenal. "Catholic Christendom, 1300-16oo", Leiden-Boston, Brill, 2016, xii + 463 p., con introduzione della Curatrice e contributi di A.G. Beaver, F. Rodríguez Mediano, V. López Fadul, C. Vincent-Cassy, P.M. Tommasino, R. Szpiech, T. Soto e K.K. Starczewska, G.A. Wiegers, J.J. Fowler, S. Pastore, M. García-Arenal, S. Kimmel, F. Pereda.

46 El Rouayheb, K. Islamic Intellectual History in the Seventeenth Century. Scholarly Currents in the Ottoman Empire and the Maghreb. New York, Cambridge University Press, 2015, xvi $+399 \mathrm{p}$. 
imitazione, bensì vide linee di sviluppo intellettuale e culturale troppo spesso misconosciute o ignorate. Per tale ragione, accettandone i risultati, non si può che collocare il saggio tra quelli dedicati all'analisi delle realtà precontemporanee del mondo islamico che ne prepararono i grandi cambiamenti. Il tema è l'Impero ottomano e la sua storia intellettuale, soprattutto letta attraverso la tradizione di studio e rilettura delle scienze razionali come attestate dalla circolazione manoscritta e dai network di studiosi attivi. L'Autore dimostra come visioni riduttrici e valutazioni stereotipate di decadenza culturale non collimano con le testimonianze manoscritte e linee di sviluppo di pensiero che furono tutt'altro che mera imitazione dell'eredità precedente. Il libro sviluppa tale concetto in varie direttrici, evidenziando i rapporti tra tradizione intellettuale e scienze religiose, l'evoluzione delle ādāb al-baht, l'emergere della lettura personale come pratica culturale nuova, la condanna del taqlïd (imitazione) e la natura delle controversie teologiche influenzate da sapere razionale, e quindi l'attitudine delle tendenze mistiche di ispirazione akbariana connesse al concetto della wahdat al-wuğüd. Tutto viene discusso evidenziando mobilità di studiosi e la circolazione delle idee e del dibattito intellettuale, da Maghreb a Persia e passando per i contributi più svariati in una vicenda culturale in cui prevalgono elementi di unità nella diversità di interpretazioni e letture. Se da più parti e da anni si lamenta la necessità di rileggere in modo nuovo tanti capitoli di storia intellettuale musulmana dopo il V/XI secolo, anche per superare vecchie visioni riduttive e viziate da pregiudizio, questo saggio è uno dei migliori esempi di come questo possa essere fatto: con un attento e inoppugnabile scrutinio di documenti e fonti. Queste dimostrano complessità di connessione e profondità del discorso intellettuale di un ambiente che aveva ben poco da invidiare ad altri periodi di solito considerati più dinamici o più significativi nell'elaborazione del pensiero se non della storia musulmana.

L'introduzione della stampa a caratteri mobili nel mondo arabo nel corso del XIX secolo è un capitolo appassionante di storia culturale solo in parte esplorato. Hala Auji vi ha contribuito in maniera esemplare con la sua ricostruzione della storia della produzione libraria a Beirut da parte della stamperia americana, nel periodo tra 1820 e $1860 .{ }^{47}$ La collocazione del saggio in una collana dedicata alla storia dell'arte e dell'archeologia rappresenta una precisa scelta culturale, così come l'ampio corredo di riproduzioni dei primi prodotti tipografici, ma oscura in parte il portato storico-culturale dell'analisi

Auji, H. Printing Arab Modernity. Book Culture and the American Press in NineteenthCentury Beirut, "Arts and Archeology of the Islamic World" n. 7, Leiden—Boston, Brill, $2016, \mathrm{xv}+155 \mathrm{p}$. 
che è dedicata anche ad altre tematiche che non il prodotto tipografico in sé. Questo non manca, ma sta accanto alle questioni di produzione a partire dai manoscritti, di scelte editoriali e culturali e infine di attivismo religioso cristiano nell'età della crescente presenza e invasività occidentale. L'analisi, di conseguenza, tratta del significato dell'introduzione della stampa nel mondo arabo e in Libano in particolare e dall'altro quello della presenza protestante americana che fu attiva con queste sue edizioni prodotte in loco soprattutto tra $1834 \mathrm{e}$ 1867. Gli aspetti tecnici e il tema centrale della produzione di una Bibbia a stampa protestante sono questioni centrali in tale vicenda, così come la competizione con le altre missioni e la ricaduta di tale rivalità nella realtà delle comunità cristiane libanesi e sui percorsi di evoluzione e modernizzazione della locale scena intellettuale. In quest'ultimo ambito, vengono doverosamente discusse le vicende che hanno visto la partecipazione di Buṭrus al-Bustānī (m. 1883) e Nāṣîf al-Yāziğ̀i (m. 1871).

\section{Islam contemporaneo}

Noorhaidi Hasan e Fritz Schulze hanno raccolto dodici contributi dedicati a questioni di genere e di diritti civili nel discorso giuridico islamico. ${ }^{48}$ La parte iniziale del titolo, che unisce visioni indonesiane e tedesche, sposta il tiro dall'ambito puramente scientifico a quello dello scambio tra studiosi se non trascuole di studio e analisi diverse, lontane, e unite, almeno in parte, dall'oggetto di studio. Si tratta infatti di una miscellanea di cui non è sempre facile cogliere spirito unitario oppure ragione che non sia quella di coordinare un incontro collegiale (realizzato in concreto in due conferenze tra Germania e Indonesia) in cui esporre le proprie ricerche. Non mancano tuttavia contributi interessanti soprattutto per il nucleo tematico più consistente, ovvero l'islam e organizzazioni islamiche nell'Indonesia contemporanea. Si segnala a tal riguardo il capitolo di S. Ruhaini Dzuhayatin che descrive le due organizzazioni islamiche più importanti della Muhammadiyya e della Nahdlatul Ulama, indagando nella dimensione di genere dopo averne percorso vicende nel corso del xx secolo e assetti organizzativi. Significativo e interessante è anche il profilo dei tafsìr indonesiani sul tema della poligamia di F. Schulze. In

48 Indonesian and German Views on the Islamic Legal Discourse on Gender and Civil Rights.

A cura di N. Hasan e F. Schulze. "Studies on Islamic Cultural and Intellectual History" n. 1, Wiesbaden, Harrassowitz, 2015, 199 p., con introduzione dei Curatori e contributi di H. Ilyas, S. Hasyim, S. Ruhaini Dzuhayatin, F. Schulze, N. Hasan, M. Afandi, Saifuddin (sic),

C. Derichs, I. Schneider, I. Gallala-Arndt, F. Wapler, G. Duttge. 
generale divorzio, legge matrimoniale, apostasia e altri temi vengono affrontati negli altri capitoli sull'Indonesia tra cui segnaliamo, per ultimo, quello di C. Derichs su una controversia intorno all'uso del termine Allāh in una pubblicazione islamica nel $2008 \mathrm{e}$ le polemiche conseguenti. Gli ultimi capitoli abbandonano l'Indonesia e trattano rispettivamente di questioni di genere in Iran (I. Schneider); statuto personale in Iran (I. Gallala-Arndt); corti tedesche e legge islamica sul divorzio (F. Wapler).

Lo šayh al-Ša'rāwī (m. 1998) è stato senza alcun dubbio un grande protagonista dell'islam contemporaneo, soprattutto in Egitto. Sebbene non influente, dal punto di vista teoretico, come al-Qaraḍāwī, ne rappresenta per certi versi un alter ego più significativo in quanto fenomeno sociale. Jacquelene G. Brinton gli ha dedicato uno studio a quasi vent'anni della sua morte. ${ }^{49} \mathrm{Il}$ saggio affronta molte questioni (vita, rapporto con società egiziana, con ulema e al-Azhar e quindi la costruzione della sua autorità religiosa attraverso linguaggio, televisione e l'elaborazione personale di alcune tematiche islamiche) ed è generalmente ben documentato, anche se ignora sistematicamente quel che è stato scritto su al-Ša'rāwī non in lingua inglese. Il profilo offerto è comunque ben costruito e restituisce vicende e attitudini di una figura centrale sulla scena egiziana degli ultimi decenni del secolo scorso. Dove però sorge più di un dubbio, è nella collocazione e anche nel giudizio della sua attività, sostanzialmente di predicatore ma soprattutto esegeta. L'Autrice sembra più attenta a non toccare questa parte fondamentale dell'attività di al-Ša'rawī e astenersi da un giudizio anche storico per privilegiare l'impatto sociale della sua figura. La scelta è deliberata, così come quella di tacere sugli aspetti più problematici del suo ruolo politico sulla scena egiziana. Così facendo si evita, colpevolmente, di entrare nel merito della evidente banalizzazione del discorso esegetico prodotta da al-Ša'rāwī, indotta senz'altro dal mezzo televisivo, e che riflette un percorso culturale di grande rilievo nella storia dell'islam contemporaneo. Ignorare ciò, oltre a guastare un saggio che ha molti meriti, è altamente rischioso oltre che mistificatorio. Astenersi dal giudizio non rende alcun servizio all'indagine, ma solo all'onda lunga di un certo anti-orientalismo, e implica un sostanziale abdicare dal ruolo dello studioso, soprattutto quando, come in questo caso, si possiedono tutti gli elementi per tentare una piena comprensione di una figura ambigua e complessa, senza tacerne nulla.

Quel che pensano gli ulema wahhabiti sauditi dello sciismo in genere è un aspetto importante nella realtà islamica contemporanea. Lo studio sull'argomento di Raihan Ismail è un'analisi documentata e puntuale delle variegate 
posizioni saudite nei confronti delle diverse forme di sciismo e della presenza sciita sia allinterno del regno sia nelle nazioni circostanti. ${ }^{50}$ La base documentale è costituita dalla letteratura prodotta, dalle fatwà emesse nel corso degli ultimi decenni e da una profondità storica che rintraccia fino al fondatore Muhammad b. 'Abd al-Wahhāb l'origine dell'atteggiamento wahhabita. Oltre a ciò, la lettura della realtà politica saudita e degli schieramenti degli stessi ulema su posizioni diverse mette in luce come nel rapporto con le altre forme di religiosità islamica, la sommaria divisione tra ulema tradizionalisti e, relativamente, "progressisti" non funziona. Il saggio procede quindi a individuare le peculiarità dello schieramento saudita, un profilo storico della divisione sunniti-sciiti, la critica teologica wahhabita contro lo sciismo, e infine i rapporti verso la minoranza sciita interna e verso l'Iran e gli altri paesi (Iraq, Bahrein e Yemen) con forte presenza sciita. Il volume si basa su un costante e puntuale riferimento a fonti lette con attenzione e con piena capacità di lettura che sa andare oltre le posizioni teologiche, e leggervi i posizionamenti politici dettati dalla casa regnante. Per quanto riguarda più direttamente la questione storico-religiosa, le posizioni degli ulema sauditi sono comunque contrassegnate da un'avversione che è anche il frutto di una realtà che vede lo sciismo sempre più presente nella realtà politica del Vicino Oriente. Benché il riferimento sia Ibn Taymiyya, certe posizioni liquidatorie vanno oltre e dimostrano come la realtà complessa soprattutto di Iraq, ma anche di Bahrein, Yemen e della minoranza interna siano un fattore determinante.

Benché di Islam non si parli mai, la storia della comunità ebraica di Baghdad nella prima metà del xx secolo ricostruita da Aline Schlaepfer è importante per le vicende dell'arabismo e per la storia politica di una delle nazioni chiave del mondo arabo e islamico come l'Iraq. ${ }^{51}$ La comunità ebraica di Baghdad costituiva a inizio secolo quasi un terzo della popolazione e le sue vicende anche di partecipazione politica accompagnano nascita e sviluppi della nazione irachena, dalla fine della presenza ottomana e l'emergere dei nazionalismi al panarabismo e, a cavallo della Seconda Guerra Mondiale, al crescere dell'influenza comunista e sionista. La rinascita (nahḍh) vi ha pure un ruolo, così come la questione della partecipazione ebraica alla sfera pubblica e alla vita culturale.

La sacralizzazione o islamizzazione dello spazio, attraverso la rappresentazione urbanistica o architettonica di emblemi o segni "islamici" è fenomeno storico, ma nella realtà contemporanea ha sovente accompagnato le politiche di re-islamizzazione e riaffermazione di una "visibilità" tangibile dell'islam

$50 \quad$ Ismail, R. Saudi Clerics and Shĩa Islam. New York, Oxford University Press, 2016, xv + 309 p.

51 Schlaepfer, A. Les intellectuelles juifs de Bagdad. Discours et allégeances (1908-1951). "Christians and Jews in Muslim Societies" n. 3, Leiden-Boston, Brill, 2016, xi + 318 p. 
nello spazio sociale. Alla situazione libanese in tale ambito, nello specifico nelle politiche di Rafìq al-Ḥarīīì è dedicato lo studio di Ward Vloeberghs. ${ }^{52} \mathrm{La}$ costruzione di moschee, come il caso indicato ad inizio di questa ricerca, ovvero la prima pietra della moschea Muhammad al-Amīn posta il primo giorno di Ramadan 1423 (6 November 2002) da Ḥarīī e dal Muftì Qabbānī, vi ha un ruolo centrale. Benché radicato, come si afferma preliminarmente, nelle scienze politiche avendo come focus primario le nozioni di potere e visibilità, è inevitabile che altre discipline convergano nel definire un tema che riguarda la più ampia questione della legittimazione da parte di ogni forma di potere. L'architettura nello spazio pubblico nasce proprio da questa esigenza. L'originalità del saggio sta perciò nel percorso che viene indagato e nella retorica di realizzazione da parte dei protagonisti, in cui lo svelamento del progetto politico è inevitabile ma che nella descrizione dettagliata appare ancora più evidente. Questo non può che passare per un'analisi dettagliata della storia personale di Harīî̄, i suoi riferimenti culturali e le sue conseguenti scelte politiche, funzionali a un progetto che riflette ciò ma anche altre contingenze, oltre a quanto dettato dal momento politico. E nel caso della costruzione di moschee, al di là della storia singola e personale, è indubbio che la storia degli ultimi decenni ne ha registrato una crescita esponenziale e di proporzioni mai viste, in nome di una monumentalizzazione, spinta da attori diversi e spesso in competizione, che riflette esigenze di vario tipo.

\section{Islam politico e radicale}

Beverly Milton-Edwards ha scritto un profilo introduttivo sulle vicende della Fratellanza Musulmana in Egitto, Palestina, Giordania, Tunisia, Yemen e nella sua organizzazione transnazionale. ${ }^{53} \mathrm{Al}$ di là del richiamo alla più recente attualità e quindi alle primavere arabe e, di conseguenza, alla breve stagione di potere in Egitto con la presidenza Morsi, il saggio fa molto di più: dopo un capitolo introduttivo sull'origine del movimento e la sua organizzazione nel corso della sua storia quasi secolare, si prendono in esami i casi nazionali più significativi del mondo arabo, anche qui delineando una lettura storica che riesce a dare un quadro completo e accessibile. In questa prospettiva sono soprattutto

$5^{2}$ Vloeberghs, W. Architecture, Power and Religion in Lebanon. Rafiq Hariri and the Politics of Sacred Space in Beirut. "Social, Economic, and Political Studies of the Middle East and Asia" n. 114, Leiden-Boston, Brill, 2016, xiii + 463 p.

53 Milton-Edwards, B. The Muslim Brotherhood. The Arab Spring and its Future Face. London-New York, Routledge, 2016, $\mathrm{x}+234 \mathrm{p}$. 
le logiche politiche quelle privilegiate, e non potrebbe essere altrimenti, per poterle valutare pienamente nello specifico nazionale e anche negli esiti diversi tra Egitto, Palestina e Giordania, ad esempio, oppure nelle realtà, per motivi opposti, tra loro assai diverse. Il capitolo sull'organizzazione transnazionale allarga la panoramica soprattutto all'Occidente e alla diffusione anche qui di organizzazioni parallele alla Fratellanza. Nella sostanza si tratta di un saggio che combina profili informativi utili a analisi condivisibili sulla situazione attuale, del quale non si può che condividere un sostanziale pessimismo sulla capacità delle varie organizzazioni di esercitare un ruolo politico attivo dopo la breve stagione delle primavere arabe, frutto dell'incapacità politica di mediare obiettivi e strategie con altre forze politiche. Inoltre, l'inaridirsi e il ridursi di possibili alleati, ora praticamente solo Qatar e Turchia, rendono le possibilità di manovra alquanto ridotte.

Le vicende dell'Isis hanno cominciato ad attirare l'attenzione di ricercatori e analisti e non solo quella di giornalisti e autori di instant book. Jack Covarrubias, Tom Lansford e Robert J. Pauly Jr. hanno raccolto una serie di saggi che ne analizzano ideologia e azione. ${ }^{54}$ Le tre parti in cui è diviso il volume trattano in ordine delle origini dello Stato Islamico, del significato della sua presenza nell'ambito regionale del Vicino Oriente e del Golfo, e quindi del rapporto tra Stato Islamico e Occidente. Benché si tratti di una raccolta senza alcun dubbio utile e che traccia alcune linee condivisibili dal punto di vista politico di ciò che rappresenta la nascita di questa realtà tra Siria e Iraq, il volume nel suo complesso affronta soprattutto le questioni politiche connesse alla realtà regionale e alla percezione occidentale del fenomeno. La tematica più propriamente ideologica è di fatto lasciata in secondo piano, e quella islamistica ancor di più. Il quadro storico della regione all'origine dell'instabilità e quindi della nascita dello Stato Islamico lascia la scena alla questione della sicurezza, sottolineando come ancora una volta l'analisi nel suo insieme riflette il problema dell'impatto sulla percezione occidentale della realtà vicino-orientale. Nulla di male in ciò, ma la capacità di guardare oltre queste coordinate è forse ciò che ne permetterebbe una più efficace comprensione ed eviterebbe il rischio di farne un'entità significativa in quanto contrapposta all'"ordine" occidentale, sostituendo in ciò al-Qaeda. Pauly Jr., R. Yon, P.R. Dean, R. Alan King, M, Sedgwick, C. Carlee, J. Brown, R. Yon e J.L. Long Jr., R.J. Pauly Jr. e K. Roberts, B. Carriere, D. Conduct D. Malet e L. West, R.J. Pauly Jr. 
Nell'ambito della storia dell'islam politico e radicale, il Sudan ha senz'altro un ruolo speciale. La fondazione di uno stato islamico, le vicende controverse relative ad al-Tūrābī e quindi la divisione più recente dal Sud Sudan sono tutti fattori significativi oltre che dal punto di vista storico, anche da quello della storia religiosa del paese. Noah Salomon ne propone una lettura che egli stesso definisce etnografica, anche se cerca di abbracciare approcci diversi. ${ }^{55}$ Il tema generale è quello del rapporto tra islam istituzionale dello stato islamico sudanese e sfera pubblica religiosa di mediazione tra spinte istituzionali e appartenenza religiosa dettata da altre istanze, ad esempio l'appartenenza agli ordini sufi. Tutto ciò viene ricostruito nelle dinamiche storiche di lungo corso, dettate da tendenze già innestate dalla presenza coloniale e nella sostanza continuate dal regime islamista iniziato nel 1989. In tale situazione il saggio ricostruisce comunque i processi di riforma tentati e perseguiti, le dinamiche di definizione di una religiosità e moralità individuale nella realtà contemporanea e soprattutto nella realtà cittadina di Khartoum. Il risultato finale è un percorso di analisi della parabola di uno stato islamico, letto nella dialettica tra volontà di definizione religiosa e costruzione pratica di una società non sempre in linea con questi, anche se ricca di varianti e di percorsi tradizionali. Apportando una serie di testimonianze utili, lo studio, nel suo complesso, rappresenta una significativa riflessione sui modelli dell'islam politico contemporaneo. Se dimostrarne la meno significativa incidenza del confronto con i processi di laicizzazione e di occidentalizzazione non è così agevole, si tratta comunque di un utile sguardo su come, in tale ambito, le evoluzioni interne, anche di lunga data, siano non meno importanti dei proclamati intenti di rottura e riforma.

\section{Islam e altre religioni}

La storia delle traduzioni in arabo della Bibbia sono un capitolo importante dei rapporti tra mondo arabo, a maggioranza musulmana, e testi sacri cristiani. David D. Grafton discute la storia della traduzione araba apparsa nel 1865 a Beirut, ad opera delle missioni evangeliche statunitensi e nota come Bibbia Van Dyke. ${ }^{56}$ Il saggio ripercorre questa storia, avvalendosi anche delle testimonianze manoscritte finora poco considerate, per comprendere meglio genesi e percorso di una traduzione in arabo giustamente famosa e che avvenne in un

\footnotetext{
55 Salomon, N. For Love of the Prophet. An Ethnography of Sudan's Islamic State. PrincetonOxford, Princeton University Press, 2016, xvii + 242 p.

56 Grafton, D.D. The Contested Origins of the 1865 Arabic Bible. "History of Christian-Muslim Relations" n. 26, Leiden-Boston, Brill, 2015, xiii + 275 p.
} 
secolo di grandi fervori cristiani. Particolare attenzione è data alla ricostruzione biografica delle cinque persone coinvolte il cui contributo viene discusso in dettaglio: Eli Smith, Buțrus al-Busțānī, Nāṣif al-Yāziğì, Cornelius Van Dyck e Yūsuf al-Aṣîr. Vi ritroviamo storia delle missioni protestanti nordamericane, le loro evoluzioni a contatto con Vicino Oriente islamico e con le comunità cristiane orientali, ma anche il ruolo complesso e a volte ambiguo verso un'operazione come questa di una figura importante per la scena culturale libanese come Buțrus al-Busțānī. Molte le questioni trattate, dalla natura e scopi della missione protestante americana tra scuole e stamperia, all'analisi della ricezione della Bibbia in studi, come ad esempio quelli di Albert Hourani ed altri in relazione alla nahdah. Il rapporto con traduzioni cattoliche non viene trascurato prima della seconda parte del saggio che analizza nello specifico il lavoro di Van Dyck che intervenne su quello iniziale di Smith, più eclettico e quindi problematico in relazione al textus receptus, da cui si discostava per desiderio di precisione e anche per effetto dei prodotti del rinnovato interesse critico-testuale per la Bibbia del XIX secolo. Tutto ciò, va sottolineato, è basato sull'uso e lo studio dei sessantanove manoscritti che sopravvivono dell'impresa e che raccolgono i contributi diversi e il lungo processo di revisione.

I volumi della serie Christian-Muslim Relations. A Bibliographical History sono destinati a divenire un imprescindibile punto di riferimento per gli studi sui rapporti tra Islam e cristianesimo così come si è sviluppato storicamente, e quindi soprattutto nel confronto polemico. La produzione e stampa dei volumi della serie, nonostante la complessità, procede, per fortuna, celermente ed è giunta ora all'ottavo volume dedicato al XVII secolo in nord Europa. ${ }^{57}$ Isole britanniche e Olanda sono le aree più ricche di produzioni relative al mondo arabo e quindi all'islam, ma tale opera risulta ancor più preziosa per la segnalazione delle non meno numerose e significative produzioni polacche o dall'Europa orientale che si dimostrano una condizione altrettanto variegata e in genere maggiormente informata sulla realtà dell'Impero ottomano. Infine, nel loro complesso, elementi di continuità ma anche novità caratterizzano autori e opere qui analizzati con quelle del secolo precedente, all'insegna di un confronto polemico che fu ancora, fino alla fine del secolo e sostanzialmente all'assedio di Vienna del 1683, una contrapposizione militare, culturale e religiosa viva e drammatica.

Christian-Muslim Relations. A Bibliographical History. Volume 8. Northern and Eastern Europe (160o-170o). A cura di D. Thomas e J. Chesworth, con C. Bennet, L. Demiri, M. Fredericks, S. Grodź e D. Pratt. "History of Christian-Muslim Relations" n. 29, LeidenBoston, Brill, 2016, xiii + 1018 p., con contributi, oltre alle schede bio-bibliografiche, di J.-A. Ezra, K. Steenbrink. 
Anche se non si tratta di un'analisi della visione islamica di altre religioni, bensì dell'occidente latino in generale, lo studio di Daniel G. König è un contributo significativo alla definizione della visione islamica dell'"altro". ${ }^{8} \mathrm{Si}$ tratta innanzitutto di un saggio estremamente documentato e con costante riferimento, nelle ricche note, a fonti primarie e alla letteratura specialistica sui singoli temi trattati, come testimonia la sconfinata bibliografia (p. 349-418), e che offre consistente documentazione alla tesi di fondo, ovvero che il mondo islamico non fu disinteressato alla conoscenza dell'occidente latino per una presunta superiorità culturale. Da qui l'analisi minuziosa di fonti geografiche e storiche che descrivono e menzionano l'Occidente latino su alcune questioni significative. La storia romana, la definizione dei Visigoti, quella dei Franchi e l'evoluzione della conoscenza sul papato sono le questioni percorse attraverso una valutazione storica della diffusione di nozioni dalle prime opere a quelle fino al XV secolo. In tale percorso costante è il riferimento al luogo per eccellenza di confronto diretto e di definizione di alcune di tali questioni, ovvero la penisola iberica. Tutto ciò è affrontato anche per gli aspetti di storia letteraria, per i canali di diffusione e il passaggio di informazioni e le innumerevoli questioni di tipo linguistico che emergono dalle fonti arabe. $\mathrm{E}$ in tale quadro vengono attentamente valutate le dinamiche di cambiamento in atto a partire soprattutto dal XII secolo per effetto dell'espansionismo europeo. L'analisi non ribalta del tutto l'assunto iniziale, anche perché i dati relativi a una maggiore diffusione di notizie sul mondo islamico da parte occidentale e un maggiore interesse, ad esempio, rispetto all'India che non all'Occidente da parte islamica sono letture storiche basate su dati documentali difficilmente controvertibili. Tuttavia le fonti attestano un crescente interesse e conoscenza per quell'Occidente latino che, in fin dei conti, appariva forse poca cosa o qualcosa di ridotto, rispetto a tutto il mondo islamico e le altre regioni ai confini della dār al-islām, come India, Cina, mondo slavo o Africa nera. Gli autori musulmani erano interessati alla riva nord del Mediterraneo, e raccolsero informazioni svariate, ma nella misura in cui furono interessati anche alle altre realtà.

\section{Orientalismo}

La storia della letteratura latina medievale su Muhammad è un capitolo ampio e ancora in parte inesplorato. Fernando González Muñoz ha pubblicato,

$5^{8}$ König, D.G. Arabic-Islamic Views of the Latin West. Tracing the Emergence of Medieval Europe. Oxford, Oxford University Press, 2015, xiv +436 p. 
tradotto e commentato quattro diversi testi letterari del XII secolo. ${ }^{59} \mathrm{Si}$ tratta di quattro opere diverse per contenuti e per forma, dalla prosa alla versificazione di vario genere, grosso modo scritti a cavallo della metà del secolo, e quindi in un periodo che rimanda alla seconda crociata. Il testo a cui è dedicato maggiore spazio e che è per certi versi più significativo è senza dubbio la Vita Mahumeti di Embrico di Magonza, di cui viene ricostruita e attraversata origine e storia manoscritta. Meno spazio è dedicato agli altri testi: Otia de Machomete di Gautier di Compiègne, un capitolo tratto da Deigesta per Francos di Guiberto di Nogent e la Vita Machometi di Adelfo. Un'ampia introduzione colloca opere nel periodo storico e nella tradizione letteraria specifica, quindi vengono descritte e presentate le singole opere prima della loro riproduzione nell'originale latino con traduzione spagnola a fronte. Non si tratta di testi inediti, ma questo nulla toglie all'utilità del lavoro e del profilo che viene tracciato. Distorsioni di natura diversa accompagnano questi ritratti del Profeta, tra polemica medievale e l'emergere di attitudini diverse e più "scientifiche", come la coeva vicenda di Pietro il Venerabile attesta. La sensazione generale che emerge è ancora una volta quella della complessità e varietà letteraria e culturale e come, pure nella distorta visione polemica, stereotipi di vario tipo si creano, sopravvivono e si alternano in capitoli di una storia letteraria che non sempre permette di mostrare i precisi canali di passaggio. Nel loro complesso, inoltre, questa letteratura mostra come la "leggenda" occidentale su Muhammad abbia storia e complessità antiche e come la circolazione di tradizioni orali, di vario tipo e con finalità diverse, percorre con uguale forza la trasmissione manoscritta di opere letterarie tra loro diverse per contenuti e anche per elaborazione letteraria.

A un periodo più tardo, e in particolare all'età moderna, guardano gli studi raccolti da Andrea Celli e Davide Scotto in un numero monografico della Rivista di Storia e Letteratura Religiosa. ${ }^{60}$ Numerosi i contributi significativi e che contribuiscono alla conoscenza della percezione dell'islam nell'Europa tra XVI e XVIII secolo. Traduzioni e studi occidentali del Corano (K.K. Starczewska, Ó. De la Cruz Palma e M. Plana) si alternano all'analisi di letteratura polemica

59 González Muñoz, F. Mahometica. Ficciones poéticas latinas del siglo XII sobre Mahoma. "Nueva Roma. Bibliotheca Graeca et Latina Aevi Posterioris" n. 42, Madrid, Consejo Superior de Investigaciones Científicas, 2015, 289 p.

6o "Esperienza e rappresentazione dell'islam nell'Europa mediterranea (secoli XVI-XVIII)". A cura di A. Celli e D. Scotto. Numero monografico di Rivista di Storia e Letteratura Religiosa, 51, n. 3 (2015), p. 393-704, con introduzione dei Curatori e contributi di K.K. Starczewska, D. Scotto, E. Colombo, C. Ilham Álvarez Dopico, Ó. De la Cruz Palma e M. Plana, S. Schreiner, C. Burnett, J.C. Villaverde Amieva, A. Celli, R.F. Glei, R. Tottoli. 
o la produzione europea sull'islam (D. Scotto, E. Colombo, C. Ilham Álvarez Dopico, S. Schreiner, A. Celli). Alcuni di questi contributi e un altro ancora (C. Burnett) analizzano direttamente o indirettamente capitoli della storia degli studi orientalistici e sull'islam nell'Europa pre-contemporanea, anche alla luce dell'incidenza che in ciò ha avuto il confronto/scontro tra Europa cattolica ed Europa della Riforma (R.F. Glei, R. Tottoli). Infine, il saggio di J.C. Villaverde Amieva analizza la tradizione in Aljamiado della storia del dialogo tra Mosé e Dio (Munāğăt Mūsā) partendo da una versione prodotta da Moriscos in Tunisi, e con un'indagine nella storia della trasmissione che offre un esempio importante di come si possa indagare negli aspetti testuali della letteratura di produzione morisca. Nel suo complesso i saggi, frutto di una collaborazione fruttuosa con il Centre for the History of Arabic Studies in Europe del Warburg Institute di Londra rappresentano un contributo importante in un settore di studi che ha visto sostanziali novità negli ultimi anni. L'organizzazione del volume mostra infatti un'attenzione peculiare, e condivisibile, per le principali direttrici su cui si articola l'interesse per l'Islam: interesse di conoscenza e intento polemico, con un evidente forte prevalere della prima al di là della retorica necessità del secondo. Su tutto ciò, la realtà storica di confronto con l'Impero ottomano da un lato e lo scontro tra Europa cattolica e protestante rappresentano fattori tutt'altro che secondari.

Dopo tanta letteratura post-saidiana di critica esplicita o implicita contro l'Orientalismo, gli ultimi anni hanno visto analisi di segno diverso e in varie direzioni. ${ }^{61}$ Aaron W. Hughes in particolare ha criticamente attaccato gli studi islamici occidentali che, anche per l'influenza della critica saidiana, scivolano verso l'apologetica e abbandonano lo spirito critico che deve prevalere negli studi religiosi. L'assunto di partenza è condivisibile benché espresso in termini troppo perentori: non spetta allo studioso dell islam entrare nel merito delle divergenze e differenti visioni, né perorare visioni pseudo-confessionali o ricerche di autenticità o identità, dato che ciò pertiene alla teologia $\mathrm{e}$ non certo a una disciplina storica come l'islamistica. Il tutto, di conseguenza, verte attorno alla grossa questione del significato di un approccio interno (da simpatetico o convertito) o uno esterno-a rischio dell'imperante accusa di "fare Orientalismo", con inaccettabile confusione, secondo l'Autore, tra oggetto e soggetto. Il saggio effettivamente mette in luce un risvolto pericoloso della ricerca nordamericana soprattutto dopo l'11 settembre, una scena politica e sociale attraversata da islamofobia e da spinte di segno opposto. È tuttavia esagerato ricondurre tali limiti a tutto il settore, anche se di ciò è ben avveduto

61 Hughes, A.W. Islam and the Tyranny of Authenticity. An inquiry into Disciplinary Apologetics and Self-Deception. Sheffield-Bristol, Equinox, 2015, xix + 143 p. 
l'Autore quando dichiara di dimostrare come dall'uso selettivo degli esempi addotti si possa costruire un discorso solido e funzionale. La scelta di analizzare la produzione scientifica o pseudo-tale di studiosi convertiti all'islam, che non sono la maggioranza nei dipartimenti dove vi sono insegnamenti sull'islam, rende l'argomentazione e la dimostrazione più facili, anche se non per questo meno valide. Non vi è del resto dubbio che il portato ideologico delle questioni toccate sollecita gli studi islamici in varie direzioni, con tendenze ad abbandonare dati documentali e filologici e sovente una ricerca di consenso interno alla comunità, anche per una correttezza politica dall'impatto inevitabile negli studi religiosi. Questa utile riflessione non sarà il ritratto di tutto il settore, eppure evidenzia i problemi che agitano il campo degli studi islamistici e quanto una ricerca critica e libera, come deve essere, faccia fatica ad eludere la questione del rapporto con oggetto di studio e i musulmani di oggi.

Aggiungiamo qui la segnalazione del volume che raccoglie sei saggi già apparsi in rivista di Miguel Ángel Ladero Quesada e accomunati dal tema generale dei rapporti tra Europa medievale e mondo islamico. ${ }^{62}$ Gli argomenti toccati vanno dalle generiche strutture politiche e sociali dei due mondi e l'immagine dell'altro (musulmano) nell'immaginario europeo cristiano, fino alle relazioni economiche tra secc. XIII e XVIIII), alcune considerazioni sulla frontiera con l'emirato di Granada e alla percezione in Castiglia del mondo islamico durante le fasi finali del Medioevo. Chiude il volume un capitolo dedicato ai Mudéjar castigliani prima di un'ampia bibliografia finale. La raccolta ha il merito di evidenziare linee comuni e coerenza di ricerche storiche che non risultano particolarmente significative per le tematiche care a questo bollettino.

\section{Islam in occidente}

La raccolta di saggi a cura di Erkan Toğuşlu discute aspetti della vita quotidiana dei musulmani europei. ${ }^{63}$ L'approccio è di tipo sociologico e nella maggior parte dei casi è impiegato per indagini di campo condotte su un campione di musulmani di varie realtà europee. Le tematiche affrontate riguardano soprattutto pratiche alimentari, i percorsi di individualizzazione che rifletterebbero

\footnotetext{
62 Ladero Quesada, M.Á. Europa medieval y mundo íslamico. Seis Estudios. Madrid, Editorial Dykinson, 2015, $256 \mathrm{p}$.

63 Everyday Life Practices of Muslims in Europe. A cura di E. Toğuşlu. "Current Issues in Islam", Leuven, Leuven University Press, 2015, 233 p., con contributi di T. Sunier, E. Mescoli, R. Brown, V. Fedele, J. Jevtic, M. El-Bachouti, L. Sterckx, W. Peumans, S. Ulu Sametoğlu, D. Guidi, A. Hussain, O. Hegazy, E. Toğuşlu.
} 
l'influenza delle realtà occidentali per ciò che riguarda tempo libero e altre attività, e il contributo di musulmani in ambito artistico o di organizzazione dello spazio, ad esempio attraverso le moschee. Non dubitiamo che la fotografia che molti contributi offrono possa rappresentare alcune realtà europee, anche se l'applicazione di modelli interpretativi su una base di ricerca a volte fatta di qualche decina di interviste induce a più di un dubbio. Inoltre, allo stesso tempo, ci permettiamo di dubitare che la rappresentazione di sé che danno gli stessi musulmani possa rappresentare un'attendibile analisi di questa realtà. Si tratta piuttosto di ciò che i musulmani dicono di loro stessi nella realtà europea e nulla di più. Detto questo, è inevitabile che in molti contributi si respiri una certa ingenuità, solidamente ed esplicitamente rafforzata dalla presunzione che questo, sociologicamente connotato, possa essere l'unico possibile e veridico approccio allo studio dei musulmani e quindi dell'Islam, contrapponendolo alla tanto avversata narrazione orientalistica (magari senza conoscerla affatto e perpetuando stereotipi e retoriche illudendosi di smascherarne altre, vd. ad es. p. 57) l'illusorietà di teorie e di ipotesi di lavoro.

La conversione all'islam da parte di occidentali rappresenta un capitolo significativo della presenza di comunità islamiche in Europa e Stati Uniti. Vari studi si sono occupati di aspetti diversi della questione e il più recente, di Amélie Puzenat, affronta in particolare la condizione femminile nell'ambito dei matrimoni misti e dei rapporti familiari in Francia. ${ }^{64}$ L'analisi è frutto di una ricerca di campo svolta dal 2006 al 2009 e quindi lontana dagli avvenimenti più recenti e ben prima delle crisi innestate dalle cosiddette primavere arabe. Il saggio tuttavia non si sottrae alla sfida della complessità e delle problematiche connesse ai rapporti tra le convertite, le nuove famiglie e quelle di provenienza e come tutto ciò implichi una ridiscussione della propria identità, che passa necessariamente anche per l'utilizzo di espedienti o elementi di visibilità nello spazio sociale. Non stupisce, in tale ottica, che largo spazio sia dedicato alle problematiche delle coppie miste e ai problemi che esse devono fronteggiare, anche in relazione alla questione educativa dei figli, accanto alla inevitabile discussione del velo, come forma di appropriazione della nuova identità e di intervento sul terreno del suo valore simbolico. L'analisi, per tutte le questioni che vengono affrontate nei nove capitoli, mette in luce la complessità dei confronti/scontri nello spazio familiare e verso l'esterno, oltre che le evidenti forme di ibridazione che sono frutto di una realtà occidentale. La consapevolezza e pienezza del discorso identitario, anche se complessa e

64 Puzenat, A. Conversions à l'islam. Unions et séparations. "Sciences des religions", Rennes, Presses universitaires de Rennes, 2015, 262 p. 
spesso fatale per le sorti, ad esempio, dei matrimoni misti, avviene su un terreno di confronto che è tipicamente occidentale e ben poco ha a che vedere con le pratiche in uso nei paesi a maggioranza musulmana. Lo stile del saggio, più simile a una narrazione che non allo studio sociologico con inevitabili riferimenti a modelli interpretativi e ricche note, aiuta nel ritrarre una serie di profili di donne che non saranno numericamente significative in termine assoluti in Francia come nell'Europa nel suo complesso, ma rappresentano comunque un fenomeno non meno importante e emblematico nella complessa realtà religiosa europea del Xxi secolo.

Il ruolo delle missioni della Ahmadiyyah in Occidente, per diffondere la propria controversa ed "ereticale" versione dell'islam nel corso del xx secolo, è poco indagata. Ben venga, quindi, lo studio di Gerdien Jonker sulle missioni in Europa nella prima metà del '90o. ${ }^{65}$ La storia parte ovviamente con la vicenda di Mirza Ghulam Ahmad di Qadian (1837-1908), il messia fondatore del movimento e il duplice impatto che il suo messaggio ebbe tra fine '8oo e prima parte del xx secolo: grandi controversie nel mondo islamico e una attenzione considerevole a livello internazionale anche per la forte vocazione missionaria del movimento. La parte iniziale descrive peculiarità, centralità del ruolo messianico di Gesù, strutture educative e novità introdotte rispetto alla visione islamica sunnita. Lo studio si focalizza poi su Europa, Gran Bretagna ed altri paesi, ma soprattutto Germania e Berlino dove ebbe maggior successo il richiamo universalistico e pacifista prodotto dalle élite musulmane del Sudest asiatico. È in questa parte che ritroviamo l'analisi di biografie di ebrei (Leopold Weiss, ad es.) che si convertirono all'islam tra le due guerre e la storia della biblioteca della moschea di Berlino. L'Autore si spinge poi giustamente oltre. Il panorama che si apre con il secondo dopoguerra è completamente diverso sia dal punto di vista della presenza europea nel mondo islamico sia per gli inizi dell'emigrazione musulmana in Europa. E la data del 1965, anno in cui la moschea di Woking passa ad altra organizzazione islamica, simboleggia perfettamente questi cambiamenti. La parte finale del saggio ritorna poi anche in Pakistan, dove gli aderenti alla Ahmadiyya sono oggi perseguitati. Tutto ciò viene ben tracciato in un saggio che è allo stesso tempo storia di un movimento islamico o pseudo-islamico e anche il profilo di un capitolo di storia europea significativo e importante per le vicende delle comunità musulmane in Occidente.

65 Jonker, G. The Ahmadiyya Quest for Religious Progress. Missionizing Europe 1900-1965. "Muslim Minorities" n. 19, Leiden—Boston, Brill, 2016, xviii + 270 p. 


\section{Strumenti}

La pregevole impresa dell'edizione e traduzione inglese delle epistole degli Ihwān al-șafā prosegue a ritmo serrato con la pubblicazione dei libri $32-36 .{ }^{66}$ Ognuno dei Curatori ha lavorato all'edizione del testo, la traduzione, l'introduzione e il commento al testo di singoli libri: P.E. Walker (l. 32-33, 35), D. Simonowitz (1. 34), G. de Callatä̈ (l. 36). I.K. Poonawala ha invece scritto la sola parte introduttiva del libro 34. Queste quattro epistole costituiscono la prima parte della sezione dedicata alle scienze dell'anima e dell'intelletto. Gli argomenti specifici sono relativi a temi filosofici di matrice ellenica e, quindi, la loro ricezione nel sistema degli Ihwān al-șafā. Qualche differenza caratterizza le quattro introduzioni, da quelle di P.E. Walker di natura soprattutto testuale, a quella di G. de Callataÿ dedicata essenzialmente ai contenuti, per finire con I.K. Poonawala che si tiene a metà strada.

Dopo qualche anno, la prestigiosa serie dedicata ai manoscritti orientali nelle biblioteche tedesche pubblica due volumi dedicati rispettivamente a quelli arabi e a quelli islamici. Rosemarie Quiring-Zoche continua la descrizione delle nuove acquisizioni di manoscritti arabi presso il prestigioso fondo della Staatsbibliothek di Berlin. ${ }^{67} \mathrm{Il}$ volume aggiunge alle precedenti pubblicazioni già disponibili la descrizione di 243 manoscritti che raccolgono 444 opere. Le descrizioni sono date in capitoli dedicati ai vari generi di letteratura, mentre la breve introduzione specifica alcune delle opere più significative per datazione o aspetti codicologici. Senza entrare nel dettaglio di quanto descritto, l'aspetto forse più significativo è la ricchezza della descrizione codicologica, che in molti casi diventa un profilo di storia del manoscritto in questione. Manfred Götz ha invece esaminato 209 manoscritti persiani e turchi conservati presso la Bayerische Staatsbibliothek di Monaco di Baviera, per un totale di 255 opere, con particolare attenzione alla descrizione dei contenuti o delle miniature che sono presenti in alcuni manoscritti, come esemplificato da alcune

66 Epistles of the Brethren of Purity. Sciences of the Soul and Intellect. Part I. An Arabic Critical Edition and English Translation of Epistles 32-36. A cura di P.E. Walker, I.K. Poonawala, D. Simonowitz, G. de Callataÿ, Oxford—New York, Oxford University Press in association with The Institute of Ismaili Studies, 2015, xxiii + 270 + 202 (in ar.) p.

67 Quiring-Zoche, R. Arabische Handschriften. Teil 7. Arabische Handschriften der Staatsbibliothek zu Berlin. Preussischer Kulturbesitz. "Verzeichnis der orientalischen Handschriften in Deutschland", XVII, B, 7, Stuttgart, Franz Steiner Verlag, 2015, xxix + $489 \mathrm{p}$. 
riproduzioni finali. ${ }^{68}$ Nel complesso si tratta di un catalogo che prosegue la tradizione della prestigiosa collana, con schedature esaustive che non tralasciano né aspetti codicologici né le opere e il loro contenuti nella storia letteraria del mondo arabo, persiano e turco.

Verena Klemm è invece la curatrice finale del catalogo del fondo di manoscritti della Rifāiiyyah portati a Lipsia a metà '80o da Johann Gottfried Wetzstein (1815-1905). ${ }^{69} \mathrm{Si}$ tratta di ben 489 manoscritti che sono brevemente descritti in base ai contenuti e quindi in un approssimato ordine tematico. L'introduzione ripercorre brevemente storia e consistenza del fondo e quindi riporta le corrispondenze con la numerazione data da Karl Vollers (m. 1909) che ne aveva curato una prima descrizione. Le schede delle singole opere contenute nei manoscritti sono tuttavia alquanto scarne, costituendo più un inventario utile a conoscere la consistenza del fondo nel suo complesso. Ł̀ senz'altro un utile strumento per una prima ricerca, ma non certo un catalogo secondo i più recenti criteri di analisi che dedicano ampio spazio anche alla descrizione del supporto e agli aspetti materiali dei manoscritti. Questi dati e la storia del fondo nel suo complesso sono invece indagati da Boriz Liebrenz in un saggio ben documentato e che ricostruisce una scena culturale complessa e assai dinamica. ${ }^{70}$ La biblioteca Rifāiiyyah, come già detto, fu acquisita da Wetzstein, in particolare quando era console prussiano a Damasco, e da qui finì poi nella collezione dell'Università di Lipsia. La biblioteca rappresenta oggi nel suo complesso una fotografia ideale degli interessi e dei percorsi educativi e di formazione e la sua stessa composizione, di conseguenza, è una testimonianza culturale di prim'ordine in tal senso. L'introduzione evidenzia i vari motivi di interesse anche attraverso la necessaria attenzione ad ambiti disciplinari e i più recenti sviluppi nello studio della cultura del libro e di bibliografia e quindi sulle difficoltà di ricavare da ciò notizie sulle pratica di lettura, anche se molti manoscritti arabi contengono alcuni informazioni su ciò. Lo studio percorre tutte le fasi di questa storia, dall'acquisto del fondo, alle sue vicissitudini

68 Götz, M. Islamische Handschriften. Teil 2. Persische und türkische Handschriften der Bayerischen Staatsbibliothek München. "Verzeichnis der orientalischen Handschriften in Deutschland", XXXVII, 2, Stuttgart, Franz Steiner Verlag, 2015, Xv + 424 p.

69 Katalog der Handschriften der Universitätsbibliothek Leipzig. A cura di V. Klemm, in coll. con B. Weismüller ed elaborazione successiva di S. Hanstein. Neue Folge. Band IV. Die Rifāīya (Refaiya). "Katalog einer Privatbibliothek aus dem osmanischen Damaskus in der Universitätsbibliothek Leipzig", Wiesbaden, Harrassowitz Verlag, 2016, lxi + 397 p.

$70 \quad$ Liebrenz, B. Die Rifāiàya aus Damaskus. Eine Privatbibliothek im osmanischen Syrien und ihr kulturelles Umfeld. "Islamic Manuscripts and Books" n. 10, Leiden-Boston, Brill, 2016, $\mathrm{xvi}+421 \mathrm{p}$. 
successive e tutti i dati desumibili dai manoscritti relativi all'uso, possesso e circolazione di questi manoscritti, ricavandone uno spaccato che dimostra al di là di ogni dubbio come il manufatto manoscritto sia una miniera di informazioni ben oltre il testo contenuto. Non si dimentica neppure ogni aspetto relativo a questi volumi, a partire dalle opere conservate, i generi attestati, i loro autori, e il significato culturale di questi dati raccolti e analizzati. Il tutto condotto con costante richiamo anche ad altre collezioni ed altri materiali che aiutino a comprendere le peculiarità della biblioteca presa in esame.

Il catalogo dei manoscritti arabi dell'Accademia Ungherese delle Scienze di Budapest si deve invece a Kinga Dévényi. ${ }^{71}$ La raccolta presentata è sicuramente interessante anche dal punto di vista storico oltre che documentale, in quanto include soprattutto manoscritti lasciati dagli Ottomani alla fine della loro occupazione dell'Ungheria (1541-1699), insieme ad altri raccolti dalle piccole comunità musulmane rimaste nel $\mathrm{xx}$ secolo quando ne vennero chiusi i luoghi di culto nel 1949. Si tratta in totale di 179 manoscritti ampiamente descritti e con profusione di immagini che offrono un rapido riscontro alle descrizioni. La raccolta conferma le caratteristiche di molte altre biblioteche di periodo ottomano, con una netta prevalenza di manoscritti di argomento grammaticale o dedicati alla logica, con i commentari e le glosse più diffuse e quindi la testimonianza concreta di processi di istruzione e di diffusione del sapere assai comuni all'interno di tutto il dominio ottomano, senza soverchie differenze tra mondo arabo e regioni balcaniche.

La prestigiosa serie dedicata alla letteratura araba dell'Africa si arricchisce di due volumi che hanno per soggetto la produzione letteraria in Mauritania e nel Sahara Occidentale, compilati a cura di Charles C. Stewart. ${ }^{72}$ Questi ultimi prodotti offrono uno standard ulteriormente migliorato di quest'opera enciclopedica ricca di informazioni e che colma una lacuna nella storia della produzione in lingua araba. Tale storia letteraria è ricostruita in profili biografici in ordine alfabetico, con ampi indici che permettono raffronti incrociati ad esempio

71 Dévényi, K. Catalogue of the Arabic Manuscripts in the Hungarian Academy of Sciences. "Islamic Manuscripts and Books" n. 9, Budapest-Leiden-Boston, Library of the Hungarian Academy of Sciences-Brill, 2016, xviii + 554 p.

72 Arabic Literature of Africa. Volume 5. The Writings of Mauritania and the Western Sahara. Part 1. A cura di C.C. Stewart, con S.A. Wuld Ahmed Salim, e M. Nouhi, B. Mbengue, B.S. Hall, A.W. Ould Cheikh, "Handbook of Oriental Studies" Sec. 1. The Near and Middle East, n. 13.V/1, Leiden-Boston, Brill, 2016, $\mathrm{xxx}+827$ p.; Arabic Literature of Africa. Volume 5. The Writings of Mauritania and the Western Sahara. Part 2. A cura di C.C. Stewart, con S.A. Wuld Ahmed Salim, e M. Nouhi, B. Mbengue, B.S. Hall, A.W. Ould Cheikh, "Handbook of Oriental Studies" Sec. 1. The Near and Middle East, n. 13.V/2, Leiden-Boston, Brill, 2016, $\mathrm{xxx}+829-2054 \mathrm{p}$. 
laddove si elencano le opere di commento e glosse. Ben 1874 sono gli autori elencati con riferimenti bibliografici e liste delle opere prodotte, che sono circa diecimila, testimoniate soprattutto in fondi manoscritti, per una storia letteraria che va dal XVII secolo fino alla seconda metà del Xx e che testimonia di una ricca attività letteraria. L'introduzione cerca di ricostruire le specificità e particolarità della regione mauritana e dell'Africa occidentale, riconducibile alla storia berbera Sanhaja e, per la storia islamica, al cosiddetto sistema educativo mahazra, forma di scuola nomade che si sviluppò a partire dal XVII secolo $\mathrm{e}$ che caratterizzò le tipologie di produzione, soprattutto su autorità religiosa, poesia e dibattiti di natura teologica. Nel complesso, di conseguenza, questo fondamentale dizionario apre una finestra inedita sulla ricchezza di un'attività intellettuale e di scrittura che dimostra la vitalità dell'educazione religiosa islamica, e della produzione conseguente. Le dinamiche non sono diverse da quelle attestate in altre realtà geografiche $\mathrm{o}$ in altri momenti storici, anche se con le specificità del caso.

La storia della circolazione dei manoscritti e dei fondi che li conservano è un aspetto essenziale per la conoscenza delle fonti primarie sulla storia della letteratura prodotta nel mondo islamico. Il discorso letto da María Jesús Viguera Molins al suo ingresso nella Accademia Reale spagnola di storia è un documentato e ricco ritratto della storia dei manoscritti arabi in Spagna. ${ }^{73} \mathrm{Il}$ ritratto offerto tratta la circolazione dei manoscritti arabi e non in al-Andalus sotto dominazione islamica, con uno sguardo al rapporto con le regioni orientali fino ai cambiamenti dettati dalle progressive conquiste e al travaso di manoscritti arabi in una situazione politica fluida. Si analizza poi la produzione locale di manoscritti arabi e il loro destino fino alla vicenda della produzione morisca in Aljamiado a cui viene dedicato largo spazio. La particolarità di tale produzione merita giustamente questa attenzione particolare soprattutto per ciò che riguarda gli aspetti più propriamente legati alla distribuzione dei centri più importanti e le particolarità di questi manoscritti in arabo. Lultima parte, infine, elenca con puntuale informazione storica su origine e sviluppi, tutti i fondi che contengono manoscritti arabi in Spagna, città per città. La descrizione data e le fonti utilizzate e citate fanno di questo "discorso" la più utile descrizione dei manoscritti arabi prodotti e conservati nella penisola iberica, e una pregevole introduzione ai vari aspetti della loro storia che possono interessare

73 Viguera Molins, M.J.. Los manuscritos árabes en España: su historia y la Historia. Discurso leído el día 28 de febrero de 2016 en el acto de su recepción pública por la Excma. Sr.a D.a M.J. Viguera Molins, y contestación por el Excmo. Sr. Don Serafín Fanjul García, Madrid, Real Academia de la Historia, 2016, 188 p. 
lo studioso dei supporti, della produzione manoscritta e della letteratura che contengono.

L'arte del manoscritto musulmano ha un significato culturale importante e fondamentale, non solo nel mondo arabo, turco o persiano, ma anche nelle altre culture musulmane estremo-orientali. Farouk Yahya ha analizzato e discusso della produzione manoscritta illustrata malese dedicata alle pratiche magiche e di divinazione. ${ }^{74}$ L'ampia ed esaustiva analisi parte giustamente dall'identificazione del campione preso in esame, costituito da 96 manoscritti Malay contenenti produzione letteraria in gran parte ancora inedita, e selezionati soprattutto in base all'apparato illustrativo incluso. I contenuti diversi dei testi e il loro rapporto con discipline correlate come l'astrologia e la fisionomica vengono definiti fin dai capitoli introduttivi, mentre all'aspetto artistico rappresentato da queste illustrazioni è poi riservata l'attenzione maggiore, con attenta ricostruzione storica nell'ambito regionale e in relazione ad altre tradizioni culturali sud-asiatiche e indiane. La parte più interessante, per i temi di questo bollettino, è dedicata alla magia nel mondo malese e all'analisi del rapporto con suggestioni e tradizioni di provenienza musulmana nella loro interazione locale. Molte le questioni coinvolte e toccate, quali la terminologia musulmana utilizzata e il sovrapporsi con pratiche divinatorie e anche sciamaniche pre-esistenti. Segue poi una ricostruzione storica della produzione manoscritta malese dal XVI secolo, prima di quella illustrata argomento dell'analisi, che è attestata solo a partire dal XVIII secolo. L'analisi materiale dei manoscritti precede infine una capitolo su committenze e modalità di trasmissione e di costruzione di autorità pseudo-religiosa collegata alle pratiche magiche e un'appendice finale che descrive tutti i manoscritti presi in esame (p. 271-295). Si segnala anche un glossario estremamente utile per i non specialisti. Nel complesso si tratta di un saggio esaustivo, ricco di suggestioni e che sa ricostruire una vicenda cultuale, storico-religiosa e artistica in maniera ottimale.

La lessicografia araba e in particolare l'organizzazione dei lemmi in dizionari è un aspetto fondamentale per l'attività di ricerca sui testi prodotti in arabo. Manfred Ullmann discute di vari aspetti connessi all'organizzazione lessicografica in un saggio che riproduce un testo trascritto di suo pugno. ${ }^{75}$ Aggiungiamo qui, inoltre, la segnalazione dell'edizione delle Mahārim al-ahlāq di al-Tַa ālibī

74 Yahya, F. Magic and Divination in Malay Illustrated Manuscripts. "Arts and Archeology of the Islamic World" n. 6, Leiden—Boston, Brill, 2016, xxviii + 349 p.

75 Ullmann, M. Theorie und Praxis der arabischen Lexicographie. Wiesbaden, Harrassowitz Verlag, 2016, $305 \mathrm{p}$. 
(d. 1039) curata da Bilal Orfali e Ramzi Baalbaki. ${ }^{76}$ L'opera, che appartiene pienamente all'adab, contiene però una parte iniziale di riferimenti a tradizioni religiose, come costume del tempo e prima di una parte preponderante dedicata ad altri aspetti di argomento etico. L'esistenza di un solo manoscritto (Leiden Or. 300), su cui si basa l'edizione, giustifica solo in parte le scelte di non descrivere l'unico testimone se non in sole cinque righe e di non accompagnare l'edizione con alcuna informazione di carattere codicologico o paleografico.

\section{Dibattiti}

La rivoluzione digitale che attraversa gli studi umanistici ha toccato da tempo anche gli studi islamici e sul Vicino Oriente in genere. Elias Muhanna ha raccolto in volume quelli che sono forse i primi contributi di riflessione sul tema, e in particolare sulle nuove possibilità offerte, i limiti e le prospettive future dell'uso di queste tecnologie negli studi di settore. ${ }^{77}$ I due contributi di T. Zadeh e D. Riedel fanno il punto della situazione su quelle che sono le principali risorse disponibili e, operando la giusta distinzione tra testi digitalizzati e cercabili in archivi e riproduzioni in Pdf, mettono in luce problemi e limiti delle possibilità di ricerca offerte. Gli altri capitoli offrono invece testimonianze di esperienze dirette, casi di studio in cui si indagano le possibilità e le auspicabili future linee di ricerca e gli strumenti che sarebbe utile ulteriormente sviluppare. È indubbio che siamo in presenza di una vera e propria rivoluzione e che questa nuova e immensa possibilità di accedere in tempi rapidi a dati in quantità inimmaginabili avrà crescente impatto sugli studi di settore, anche se alcuni percorsi proposti offrono il fianco a qualche valutazione diversa. Il volume in sé è un prodotto eccellente e finalmente mette in luce questa importante novità, ma la visione dei limiti e dei problemi elencati sembra basarsi su presupposti discutibili. L'assunto che l'era digitale segua una fase "filologica" è alquanto approssimativo, in quanto le pratiche di edizione della vecchia scuola orientalista sono state tutt'altro che fatte in dialogo con metodi critici

76 The Book of Noble Character. Critical Edition of Makārim al-akhlāq wa-mahāāin al-ād$\bar{a} b$ wa-badā'ic al-awșāj wa-gharāìb al-tashbīhāt, Attributed to Abū Manșūr al-Tha'ālibì (d. 429/1039). A cura di B. Orfali e R. Baalbaki. "Islamic History and Civilization. Studies and texts" n. 120, Leiden-Boston, Brill, 2015, 300 p.

77 The Digital Humanities and Islamic \& Middle East Studies. A cura di E. Muhanna. BerlinBoston, Walter de Gruyter, 2016, vii + 271 p., con capitolo introduttivo del Curatore e contributi di T. Zadeh, D. Riedel, C. Rossetti, N. Yaqub, M. Romanov, A. Brey, T. Grallert, J. Haro Peralta e P. Verkinden, J. Blecher, D.F. Reynolds. 
in uso in altri ambiti. Né gli studi islamistici sono stati sempre e comunque il prodotto di un lavoro che possa essere definito pienamente filologico, costante e continuo sui testi i quali, anzi, sono stati spesso sempre gli stessi e hanno in molti casi ignorato le immense biblioteche manoscritte. I nuovi data-base, in quanto tali, sono strumenti che possono aprire una nuova fase di utilizzo di testi o di sequenze testuali, se interrogati nella giusta misura e con piena consapevolezze della struttura dei prodotti letterari a cui appartengono. In una marea trionfante di scarse letture, di imperanti sforzi interpretativi che a volte non reggono l'urto dei dati documentali questi nuovi strumenti possono apparire limitati e a volte fuorvianti, ma più per pregiudizi di vario genere che per limiti veri e propri dati dalla nuova forma testuale che essi necessariamente assumono agli occhi del fruitore. 